\title{
Interfacial Heat Transport in Highly Permeable Media: A Finite Volume Approach
}

Marcelo J.S. de Lemos and Marcelo B. Saito

\section{1}

\section{Introduction}

The transport of heat inside highly permeable media has attracted the attention of scientists and engineers due to its many engineering applications. Such applications can be found in solar energy receiver devices, heat exchangers, porous combustors, grain drying equipment, heat sink units, energy recovery systems, etc. In many of these modern engineering systems the use of cellular and metallic porous foams brings the advantages of having large specific heat transfer areas, or the interfacial transport area per unit volume is large when compared with other heat-capturing devices. More realistic modeling of transport processes in such media is then essential for the reliable design and analysis of high-efficiency engineering systems.

Motivated by the wide spectrum of practical engineering applications, macroscopic transport modeling of incompressible flows in porous media has been developed over the last few decades, mostly based on the volume-average methodology for either heat [1] or mass transfer [2,3]. Classic books by Bear (1972) [4], Nield and Bejan (1992) [5] and Ingham and Pop (1998) [6], to mention a few, also document forced convection and related models for heat transport in porous media.

From the point of view of energy transfer between phases, namely the cellular material phase and the working fluid, there are basically two different models commonly found in the literature: (a) a local thermal equilibrium model and (b) a two-energy equation or thermal nonequilibrium model. The first one assumes that the bulk solid temperature does not differ much from the average value of the fluid temperature; thus local thermal equilibrium between the fluid and the solid phase is assumed. This model greatly simplifies theoretical and numerical research but the assumption of local thermal equilibrium between the fluid and the solid is inadequate for a number of practical problems [7-9]. As a result, in recent years more attention has been paid to the local thermal nonequilibrium model, both theoretically and numerically $[10,11]$. 
Accordingly, two-energy equation models have been investigated by a number of researchers. Kuwahara et al. (2001) [12] proposed a numerical procedure to determine macroscopic transport coefficients from a theoretical basis without any empiricism. They used a single unit cell and determined the interfacial heat transfer coefficient for the asymptotic case of infinite conductivity of the solid phase. Nakayama et al. (2001) [13] extended the conduction model of Hsu (1999) [14] for treating also convection in porous media. Having established the macroscopic energy equations for both phases, useful exact solutions were obtained for two fundamental heat transfer processes associated with porous media, namely steady conduction in a porous slab with internal heat generation within the solid and also thermally developing flow through a semi-infinite porous medium. Saito and de Lemos (2005) [15] considered the distribution in cell parameters and, after volume-averaging them, the interfacial heat transfer coefficient for laminar flow.

In all of the above, only laminar flow has been considered. When treating turbulent flow in porous media, however, difficulties arise due to the fact that the flow fluctuates with time and a volumetric average is applied [16]. For handling such situations, a new concept called double decomposition has been proposed for developing macroscopic models for turbulent transport in porous media [17-22]. This methodology has been extended to nonbuoyant heat transfer [23], buoyant flows [24-30], mass transfer [31] and double diffusion [32]. In addition, a general classification of models has been published [33]. Further, the problem of treating interfaces between a porous medium and a clear region, considering a diffusion-jump condition for laminar [34] and turbulence fields [35-37], has also been investigated under the concept first proposed by several authors [17-22]. Furthermore, Saito and de Lemos (2006) [38] proposed a new correlation for obtaining the interfacial heat transfer coefficient for turbulent flow in a packed bed, which is modeled as an infinite staggered array of square rods. Recently, a book has been published on the subject of turbulence modeling in porous media [39].

Motivated by the foregoing, this chapter focuses on laminar and turbulent flow through a packed bed, which represents an important configuration for efficient heat and mass transfer and suggests the use of equations governing thermal nonequilibrium involving distinct energy balances for both the solid and fluid phases. Hence, the use of such a two-energy equation model requires an extra parameter to be determined, namely the heat transfer coefficient between the fluid and the solid. This chapter reviews recent efforts in proposing correlations for obtaining the interfacial heat transfer coefficient for laminar and turbulent flow in porous material. The medium is here modeled as an infinite array of rods of distinct shapes, over which the range of Reynolds number, based on a characteristic size of the rod, is extended up to $10^{7}$.

The next sections detail the basic mathematical model, including the mean and turbulent fields for turbulent flows. Although the discussion of turbulent motion in porous media is not presented, the definitions and concepts to calculate the interfacial heat transfer coefficient for macroscopic flows are presented here. 


\section{Governing Equations}

1.2.1

\section{Microscopic Transport Equations}

Local time-averaged transport equations for incompressible fluid flow in a rigid homogeneous porous medium have already been presented in the literature and their derivation need not be repeated here [24]. The governing equations for the flow and energy for an incompressible fluid are given by

$$
\begin{aligned}
& \text { Continuity: } \nabla \cdot \mathbf{u}=0 \\
& \text { Momentum: } \rho\left[\frac{\partial \mathbf{u}}{\partial t}+\nabla \cdot(\mathbf{u u})\right]=-\nabla p+\mu \nabla^{2} \mathbf{u} \\
& \text { Energy-fluid phase: }\left(\rho c_{\mathrm{p}}\right)_{\mathrm{f}}\left\{\frac{\partial T_{\mathrm{f}}}{\partial t}+\nabla \cdot\left(\mathbf{u} T_{\mathrm{f}}\right)\right\}=\nabla \cdot\left(k_{\mathrm{f}} \nabla T_{\mathrm{f}}\right)+S_{\mathrm{f}} \\
& \text { Energy-solid phase(porous matrix): }\left(\rho c_{\mathrm{p}}\right)_{\mathrm{s}} \frac{\partial T_{\mathrm{s}}}{\partial t}=\nabla \cdot\left(k_{\mathrm{s}} \nabla T_{\mathrm{s}}\right)+S_{\mathrm{s}}
\end{aligned}
$$

where the subscripts f and s refer to fluid and solid phases, respectively. Here, $T$ is the temperature, $k_{\mathrm{f}}$ is the fluid thermal conductivity, $k_{\mathrm{s}}$ is the solid thermal conductivity, $c_{\mathrm{p}}$ is the specific heat and $S$ is the heat generation term. If there is no heat generation either in the solid or in the fluid, one further has $S_{\mathrm{f}}=S_{\mathrm{s}}=0$.

For turbulent flows the time-averaged transport equations can be written as

$$
\begin{aligned}
& \text { Continuity: } \nabla \cdot \overline{\mathbf{u}}=0 \\
& \text { Momentum: } \rho_{\mathrm{f}}[\nabla \cdot(\overline{\mathbf{u}})]=-\nabla \bar{p}+\nabla \cdot\left\{\mu\left[\nabla \overline{\mathbf{u}}+(\nabla \overline{\mathbf{u}})^{T}\right]-\rho \overline{\mathbf{u}^{\prime} \mathbf{u}^{\prime}}\right\}
\end{aligned}
$$

where the low and high Reynolds $k-\varepsilon$ models as can be used to calculate the Reynolds stresses $\rho \overline{\mathbf{u}^{\prime} \mathbf{u}^{\prime}}$ via the eddy viscosity concept, $\mu_{t}$. Equations for the turbulent kinetic energy per unit mass (TKE) and for its dissipation rate read:

Turbulent kinetic energy per unit mass (TKE):

$$
\rho_{\mathrm{f}}[\nabla \cdot(\overline{\mathbf{u}} k)]=\nabla \cdot\left[\left(\mu+\frac{\mu_{t}}{\sigma_{k}}\right) \nabla k\right]-\rho \overline{\mathbf{u}^{\prime} \mathbf{u}^{\prime}}: \nabla \overline{\mathbf{u}}-\rho \varepsilon
$$

TKE dissipation rate:

$$
\rho_{\mathrm{f}}[\nabla \cdot(\overline{\mathbf{u}} \varepsilon)]=\nabla \cdot\left[\left(\mu+\frac{\mu_{t}}{\sigma_{\varepsilon}}\right) \nabla \varepsilon\right]+\left[c_{1}\left(-\rho \overline{\mathbf{u}^{\prime} \mathbf{u}^{\prime}}: \nabla \overline{\mathbf{u}}\right)-c_{2} f_{2} \rho \varepsilon\right] \frac{\varepsilon}{k}
$$


The Reynolds stresses and the eddy viscosity are given by, respectively:

$$
\begin{aligned}
& -\rho \overline{\mathbf{u}^{\prime} \mathbf{u}^{\prime}}=\mu_{t}\left[\nabla \overline{\mathbf{u}}+(\nabla \overline{\mathbf{u}})^{T}\right]-\frac{2}{3} \rho k \mathbf{I} \\
& \mu_{t}=\rho c_{\mu} f_{\mu} \frac{k^{2}}{\varepsilon}
\end{aligned}
$$

where $\rho$ is the fluid density, $p$ is the pressure and $\mu$ represents the fluid viscosity.

In the above equation set, $\sigma_{k}, \sigma_{\varepsilon}, c_{1}, c_{2}$ and $c_{\mu}$ are dimensionless constants whereas $f_{2}$ and $f_{\mu}$ are damping functions used with the so-called low Re $k-\varepsilon$ turbulence models. The following damping functions are adopted here when computing with the low Re model:

$$
\begin{aligned}
& f_{\mu}=\left\{1-\exp \left[-\frac{(v \varepsilon)^{0.25} \gamma}{14 v}\right]\right\}^{2}\left\{1+\frac{5}{\left(k^{2} / \nu \varepsilon\right)^{0.75}} \exp \left[-\left(\frac{\left(k^{2} / \nu \varepsilon\right)}{200}\right)^{2}\right]\right\} \\
& f_{2}=\left\{1-\exp \left[-\frac{(v \varepsilon)^{0.25} \gamma}{3.1 v}\right]\right\}^{2}\left\{1-0.3 \exp \left[-\left(\frac{\left(k^{2} / \nu \varepsilon\right)}{6.5}\right)^{2}\right]\right\}
\end{aligned}
$$

where $\gamma$ is the coordinate normal to the wall. Other constants are given as $c_{\mu}=0.09$, $c_{1}=1.5, c_{2}=1.9, \sigma_{k}=1.4, \sigma_{\varepsilon}=1.3$, which were taken from Launder and Spalding (1974) [40].

Also, the time-averaged energy equations become

Energy-fluid phase:

$$
\begin{aligned}
& \left(\rho c_{\mathrm{p}}\right)_{\mathrm{f}}\left[\nabla \cdot\left(\overline{\mathbf{u}} \bar{T}_{\mathrm{f}}\right)\right]=\nabla \cdot\left(k_{\mathrm{f}} \nabla \bar{T}_{\mathrm{f}}\right)-\left(\rho c_{\mathrm{p}}\right)_{\mathrm{f}} \nabla \cdot\left(\overline{\mathbf{u}^{\prime} T_{\mathrm{f}}^{\prime}}\right) \\
& \text { Energy-solid phase(porous matrix }): \\
& \nabla \cdot\left(k_{\mathrm{s}} \nabla \bar{T}_{\mathrm{s}}\right)+S_{\mathrm{s}}=0
\end{aligned}
$$

\subsection{2}

\section{Decomposition of Flow Variables in Space and Time}

If time fluctuations of the flow properties are also considered, in addition to spatial deviations, there are two possible methodologies to follow in order to obtain macroscopic equations: (a) application of time-average operator followed by volumeaveraging [41-46], or (b) use of volume-averaging before time-averaging is applied [47-49]. However, both sets of macroscopic mass transport equations are equivalent when examined under the recently established double decomposition concept [17-22]. As mentioned above, the double decomposition concept has been published in a number of widely available journal articles [17-24], book chapters [50-53] and a 
book [39]. For the sake of completeness, a brief overview is presented here and additional details can be found in the literature cited.

Macroscopic transport equations for turbulent flow in a porous medium are obtained through the simultaneous application of time- and volume-average operators over a generic fluid property $\varphi[16]$. Such concepts are mathematically defined as

$$
\begin{aligned}
& \bar{\varphi}=\frac{1}{\Delta t} \int_{t}^{t+\Delta t} \varphi \mathrm{d} t, \quad \text { with } \quad \varphi=\bar{\varphi}+\varphi^{\prime} \\
& \langle\varphi\rangle^{i}=\frac{1}{\Delta V_{\mathrm{f}}} \int_{\Delta V_{\mathrm{f}}} \varphi \mathrm{d} V ; \quad\langle\varphi\rangle^{\mathrm{v}}=\phi\langle\varphi\rangle^{\mathrm{i}} ; \quad \phi=\frac{\Delta V_{\mathrm{f}}}{\Delta V}, \quad \text { with } \quad \phi=\langle\varphi\rangle^{\mathrm{i}}+{ }^{i} \varphi
\end{aligned}
$$

where $\Delta V_{\mathrm{f}}$ is the volume of the fluid contained in a representative elementary volume (REV) $\Delta V$ and intrinsic average and volume average are represented, respectively, by \langle\rangle$^{\mathrm{i}}$ and \langle\rangle$^{\mathrm{v}}$. Also, the left superscript $i$ represents spatial deviation.

The double decomposition idea introduced and fully described elsewhere [17-22], combines Eqs. (1.15) and (1.16) and can be summarized as

$$
\overline{\langle\varphi\rangle^{\mathrm{i}}}=\langle\bar{\varphi}\rangle^{\mathrm{i}} ; \quad i_{\bar{\varphi}}=\overline{i_{\varphi}} ; \quad\left\langle\varphi^{\prime}\right\rangle^{\mathrm{i}}=\langle\varphi\rangle^{i^{\prime}}
$$

and

$$
\left.\begin{array}{l}
\varphi^{\prime}=\left\langle\varphi^{\prime}\right\rangle^{i}+{ }^{i} \varphi^{\prime} \\
{ }^{i} \varphi=\overline{{ }^{i} \varphi}+{ }^{i} \varphi^{\prime}
\end{array}\right\} \quad \text { where } \quad{ }^{i} \varphi^{\prime}=\varphi^{\prime}-\left\langle\varphi^{\prime}\right\rangle^{\mathrm{i}}={ }^{i} \varphi-\overline{{ }^{i} \varphi}
$$

Therefore, the quantity $\varphi$ can be expressed by either

$$
\varphi=\overline{\langle\varphi\rangle^{i}}+\langle\varphi\rangle^{i^{\prime}}+\overline{{ }^{\varphi} \varphi}+{ }^{i} \varphi^{\prime}
$$

or

$$
\varphi=\langle\bar{\varphi}\rangle^{\mathrm{i}}+{ }^{i} \bar{\varphi}+\left\langle\varphi^{\prime}\right\rangle^{\mathrm{i}}+{ }^{i} \varphi^{\prime}
$$

The term ${ }^{i} \varphi^{\prime}$ can be viewed as either the temporal fluctuation of the spatial deviation or the spatial deviation of the temporal fluctuation of quantity $\varphi$ [39].

\subsection{3}

\section{Macroscopic Flow and Energy Equations}

When the average operators (1.15) and (1.16) are simultaneously applied over Eqs. (1.1) and (1.2), macroscopic equations for turbulent flow are obtained. Volume integration is performed over a REV [16,54], resulting in

$$
\text { Continuity: } \nabla \cdot \overline{\mathbf{u}}_{\mathrm{D}}=0
$$


where $\overline{\mathbf{u}}_{\mathrm{D}}=\phi\langle\overline{\mathbf{u}}\rangle^{\mathrm{i}}$ and $\langle\overline{\mathbf{u}}\rangle^{\mathrm{i}}$ identifies the intrinsic (liquid) average of the timeaveraged velocity vector $\overline{\mathbf{u}}$.

$$
\begin{aligned}
\text { Momentum: } & \rho\left[\frac{\partial \overline{\mathbf{u}}_{\mathrm{D}}}{\partial t}+\nabla \cdot\left(\frac{\overline{\mathbf{u}}_{\mathrm{D}} \overline{\mathbf{u}}_{\mathrm{D}}}{\phi}\right)\right]=-\nabla\left(\phi\langle\bar{p}\rangle^{\mathrm{i}}\right)+\mu \nabla^{2} \overline{\mathbf{u}}_{\mathrm{D}} \\
& -\nabla \cdot\left(\rho \phi\left\langle\overline{\mathbf{u}^{\prime} \mathbf{u}^{\prime}}\right\rangle^{\mathrm{i}}\right)-\left[\frac{\mu \phi}{K} \overline{\mathbf{u}}_{\mathrm{D}}+\frac{c_{\mathrm{F}} \phi \rho\left|\overline{\mathbf{u}}_{\mathrm{D}}\right| \overline{\mathbf{u}}_{\mathrm{D}}}{\sqrt{K}}\right]
\end{aligned}
$$

where the last two terms in Eq. (1.22) represent the Darcy and Forchheimer contributions [55]. Parameter $K$ is the porous medium permeability, $c_{\mathrm{F}}$ is the form drag or Forchheimer coefficient, $\langle\bar{p}\rangle^{\mathrm{i}}$ is the intrinsic average pressure of the fluid and $\phi$ is the porosity of the porous medium.

The macroscopic Reynolds stress, $-\rho \phi\left\langle\overline{\mathbf{u}^{\prime} \mathbf{u}^{\prime}}\right\rangle^{i}$, appearing in Eq. (1.22) is given as

$$
-\rho \phi\left\langle\overline{\mathbf{u}^{\prime} \mathbf{u}^{\prime}}\right\rangle^{\mathrm{i}}=\mu_{t_{\phi}} 2\langle\overline{\mathbf{D}}\rangle^{\mathrm{v}}-\frac{2}{3} \phi \rho\langle k\rangle^{\mathrm{i}} \mathbf{I}
$$

where

$$
\langle\overline{\mathbf{D}}\rangle^{\mathrm{v}}=\frac{1}{2}\left[\nabla\left(\phi\langle\overline{\mathbf{u}}\rangle^{\mathrm{i}}\right)+\left[\nabla\left(\phi\langle\overline{\mathbf{u}}\rangle^{\mathrm{i}}\right)\right]^{\mathrm{T}}\right]
$$

is the macroscopic deformation tensor, $\langle k\rangle^{\mathrm{i}}=\left\langle\overline{\mathbf{u}^{\prime} \cdot \mathbf{u}^{\prime}}\right\rangle^{\mathrm{i}} / 2$ is the intrinsic turbulent kinetic energy and $\mu_{t_{\phi}}$, is the turbulent viscosity, which is modeled in Ref. [33] similarly to the case of clear flow in the form

$$
\mu_{t_{\phi}}=\rho c_{\mu} \frac{\langle k\rangle^{i^{2}}}{\langle\varepsilon\rangle^{i}}
$$

The intrinsic turbulent kinetic energy per unit mass and its dissipation rate are governed by the following equations:

$$
\begin{aligned}
\rho\left[\frac{\partial}{\partial t}\left(\phi\langle k\rangle^{\mathrm{i}}\right)+\nabla \cdot\left(\overline{\mathbf{u}}_{\mathrm{D}}\langle k\rangle^{\mathrm{i}}\right)\right]= & \nabla \cdot\left[\left(\mu+\frac{\mu_{t_{\phi}}}{\sigma_{k}}\right) \nabla\left(\phi\langle k\rangle^{i}\right)\right]-\rho\left\langle\overline{\mathbf{u}^{\prime} \mathbf{u}^{\prime}}\right\rangle^{\mathrm{i}}: \nabla \overline{\mathbf{u}}_{\mathrm{D}} \\
& +c_{k} \rho \frac{\phi\langle k\rangle^{\mathrm{i}}\left|\overline{\mathbf{u}}_{\mathrm{D}}\right|}{\sqrt{K}}-\rho \phi\langle\varepsilon\rangle^{\mathrm{i}} \\
\rho\left[\frac{\partial}{\partial t}\left(\phi\langle\varepsilon\rangle^{\mathrm{i}}\right)+\nabla \cdot\left(\overline{\mathbf{u}}_{\mathrm{D}}\langle\varepsilon\rangle^{\mathrm{i}}\right)\right]= & \nabla \cdot\left[\left(\mu+\frac{\mu_{t_{\phi}}}{\sigma_{\varepsilon}}\right) \nabla\left(\phi\langle\varepsilon\rangle^{\mathrm{i}}\right)\right] \\
& +c_{1}\left(-\rho\left\langle\overline{\mathbf{u}^{\prime} \mathbf{u}^{\prime}}\right\rangle^{\mathrm{i}}: \nabla \overline{\mathbf{u}}_{\mathrm{D}}\right) \frac{\langle\varepsilon\rangle^{\mathrm{i}}}{\langle k\rangle^{\mathrm{i}}}+c_{2} c_{k} \rho \frac{\phi\langle\varepsilon\rangle^{\mathrm{i}}\left|\overline{\mathbf{u}}_{\mathrm{D}}\right|}{\sqrt{K}} \\
& -c_{2} \rho \phi \frac{\langle\varepsilon\rangle^{\mathrm{i}^{2}}}{\langle k\rangle^{\mathrm{i}}}
\end{aligned}
$$


where $c_{k}, c_{1}, c_{2}$ and $c_{\mu}$ are nondimensional constants. The second terms on the left-hand side of Eqs. (1.26) and (1.27) represent the generation rate of $\langle k\rangle^{i}$ and $\langle\varepsilon\rangle^{\mathrm{i}}$, respectively, due to the mean gradient of $\overline{\mathbf{u}}_{\mathrm{D}}$. The third terms in both equations are related to the generation rates due to the action of the porous matrix [18].

Similarly, macroscopic energy equations are obtained for both fluid and solid phases by applying time and volume average operators to Eqs. (1.3) and (1.4). As in the flow case, volume integration is performed over a REV, resulting in

$$
\begin{gathered}
\left(\rho c_{\mathrm{p}}\right)_{\mathrm{f}} \frac{\partial \phi\left\langle\overline{T_{\mathrm{f}}}\right\rangle^{\mathrm{i}}}{\partial t}+\left(\rho c_{\mathrm{p}}\right)_{\mathrm{f}}\left[\nabla \cdot\left\{\phi\left(\langle\overline{\mathbf{u}}\rangle^{\mathrm{i}}\left\langle\overline{T_{\mathrm{f}}}\right\rangle^{\mathrm{i}}+\left\langle{ }^{\mathrm{i}} \overline{\mathbf{u}}^{i} \overline{T_{\mathrm{f}}}\right\rangle^{\mathrm{i}}+\left\langle\overline{\mathbf{u}^{\prime} T_{\mathrm{f}}^{\prime}}\right\rangle^{\mathrm{i}}\right)\right\}\right] \\
=\nabla \cdot\left[k_{\mathrm{f}} \nabla\left(\phi\left\langle\overline{T_{\mathrm{f}}}\right\rangle^{\mathrm{i}}\right)+\frac{1}{\Delta V} \int_{A_{i}} \mathbf{n}_{\mathrm{i}} k_{\mathrm{f}} \overline{T_{\mathrm{f}}} \mathrm{d} A\right]+\frac{1}{\Delta V} \int_{A_{i}} \mathbf{n}_{\mathrm{i}} \cdot k_{\mathrm{f}} \nabla \overline{T_{\mathrm{f}}} \mathrm{d} A \\
\left(\rho c_{\mathrm{p}}\right)_{\mathrm{s}}\left\{\frac{\partial(1-\phi)\left\langle\overline{T_{\mathrm{s}}}\right\rangle^{\mathrm{i}}}{\partial t}\right\}=\nabla \cdot\left\{k_{\mathrm{s}} \nabla\left[(1-\phi)\left\langle\overline{T_{\mathrm{s}}}\right\rangle^{\mathrm{i}}\right]-\frac{1}{\Delta V} \int_{A_{i}} \mathbf{n}_{\mathrm{i}} k_{\mathrm{s}} \overline{T_{\mathrm{s}}} \mathrm{d} A\right\} \\
-\frac{1}{\Delta V} \int_{A_{i}} \mathbf{n}_{\mathrm{i}} \cdot k_{\mathrm{s}} \nabla \overline{T_{\mathrm{s}}} \mathrm{d} A
\end{gathered}
$$

where $\left\langle\overline{T_{\mathrm{s}}}\right\rangle^{\mathrm{i}}$ and $\left\langle\overline{T_{\mathrm{f}}}\right\rangle^{\mathrm{i}}$ denote the intrinsic average temperature of solid and fluid phases, respectively, $A_{\mathrm{i}}$ is the interfacial area within the REV and $\mathbf{n}_{\mathrm{i}}$ is the unit vector normal to the fluid-solid interface, pointing from the fluid towards the solid phase. Equations (1.28) and (1.29) are the macroscopic energy equations for the fluid and the porous matrix (solid), respectively.

Further, using the double decomposition concept given by Eqs. (1.17)-(1.20), Rocamora and de Lemos (2000) [23] have shown that the fourth term on the left-hand side of Eq. (1.28) can be expressed as

$$
\left\langle\overline{\left\langle\mathbf{u}^{\prime} T_{\mathrm{f}}^{\prime}\right.}\right\rangle^{\mathrm{i}}=\left\langle\overline{\left\langle\left(\left\langle\mathbf{u}^{\prime}\right\rangle^{\mathrm{i}}+{ }^{i} \mathbf{u}^{\prime}\right)\left(\left\langle T_{\mathrm{f}}^{\prime}\right\rangle^{\mathrm{i}}+{ }^{i} T_{\mathrm{f}}^{\prime}\right)\right.}\right\rangle^{\mathrm{i}}=\overline{\left\langle\mathbf{u}^{\prime}\right\rangle^{\mathrm{i}}\left\langle T_{\mathrm{f}}^{\prime}\right\rangle^{\mathrm{i}}}+\overline{\left\langle{ }^{\prime \mathbf{u}^{\prime} T_{\mathrm{f}}^{\prime}}\right\rangle^{\mathrm{i}}}
$$

Therefore, in view of Eq. (1.30), Eq. (1.28) can be rewritten as

$$
\begin{gathered}
\left.\left(\rho c_{\mathrm{p}}\right)_{\mathrm{f}}\left[\frac{\partial \phi\left\langle\overline{T_{\mathrm{f}}}\right\rangle^{\mathrm{i}}}{\partial t}+\nabla \cdot\left\{\phi\left(\langle\overline{\mathbf{u}}\rangle^{\mathrm{i}}\left\langle\overline{T_{\mathrm{f}}}\right\rangle^{\mathrm{i}}+\left\langle{ }^{i} \overline{\mathbf{u}^{i}} \overline{T_{\mathrm{f}}}\right\rangle^{\mathrm{i}}+\overline{\left\langle\mathbf{u}^{\prime}\right\rangle^{\mathrm{i}}\left\langle T_{\mathrm{f}}^{\prime}\right\rangle^{\mathrm{i}}}+\overline{\left\langle{ }^{\prime \mathbf{u}^{\prime}} T_{\mathrm{f}}^{\prime}\right.}\right\rangle^{\mathrm{i}}\right)\right\}\right] \\
=\nabla \cdot\left[k_{\mathrm{f}} \nabla\left(\phi\left\langle\overline{T_{\mathrm{f}}}\right\rangle^{\mathrm{i}}\right)+\frac{1}{\Delta V} \int_{A_{i}} \mathbf{n}_{\mathrm{i}} k_{\mathrm{f}} \overline{T_{\mathrm{f}}} \mathrm{d} A\right]+\frac{1}{\Delta V} \int_{A_{i}} \mathbf{n}_{\mathrm{i}} \cdot k_{\mathrm{f}} \nabla \overline{T_{\mathrm{f}}} \mathrm{d} A
\end{gathered}
$$


The two-energy equation model, considering a heat transfer coefficient between the fluid and solid phases, is then based on the following equations

$$
\begin{gathered}
\left(\rho c_{\mathrm{p}}\right)_{\mathrm{f}}\left[\frac{\partial \phi\left\langle\overline{T_{\mathrm{f}}}\right\rangle^{\mathrm{i}}}{\partial t}+\nabla \cdot\left\{\phi(\underbrace{\langle\overline{\mathbf{u}}\rangle^{\mathrm{i}}\left\langle\overline{T_{\mathrm{f}}}\right\rangle^{\mathrm{i}}}_{\mathrm{I}}+\underbrace{\left\langle{ }^{i} \overline{\mathbf{u}^{i} \bar{T}_{\mathrm{f}}}\right\rangle^{\mathrm{i}}}_{\text {II }}+\underbrace{\overline{\left\langle\mathbf{u}^{\prime}\right\rangle^{\mathrm{i}}\left\langle T_{\mathrm{f}}^{\prime}\right\rangle^{\mathrm{i}}}}_{\text {III }}+\underbrace{\left.\overline{\left\langle\overline{i^{\prime i} T_{\mathrm{f}}^{\prime}}\right.}\right\rangle^{\mathrm{i}}}_{\text {IV }})\right\}\right] \\
=\nabla \cdot\left[k_{\mathrm{f}} \nabla\left(\phi\left\langle\overline{T_{\mathrm{f}}}\right\rangle^{\mathrm{i}}\right)+\frac{1}{\Delta V} \int_{A_{i}} \mathbf{n}_{\mathrm{i}} k_{\mathrm{f}} \overline{T_{\mathrm{f}}} \mathrm{d} A\right]+h_{\mathrm{i}} a_{\mathrm{i}}\left(\left\langle\overline{T_{\mathrm{s}}}\right\rangle^{\mathrm{i}}-\left\langle\overline{T_{\mathrm{f}}}\right\rangle^{\mathrm{i}}\right) \\
\left(\rho c_{\mathrm{p}}\right)_{\mathrm{s}}\left\{\frac{\partial(1-\phi)\left\langle\overline{T_{\mathrm{s}}}\right\rangle^{\mathrm{i}}}{\partial t}\right\}=\nabla \cdot\left\{k_{\mathrm{s}} \nabla\left[(1-\phi)\left\langle\overline{T_{\mathrm{s}}}\right\rangle^{\mathrm{i}}\right]-\frac{1}{\Delta V} \int_{A_{i}} \mathbf{n}_{\mathrm{i}} k_{\mathrm{s}} \overline{T_{\mathrm{s}}} \mathrm{d} A\right\} \\
+h_{\mathrm{i}} a_{\mathrm{i}}\left(\left\langle\overline{T_{\mathrm{f}}}\right\rangle^{\mathrm{i}}-\left\langle\overline{T_{\mathrm{s}}}\right\rangle^{\mathrm{i}}\right)
\end{gathered}
$$

where $h_{\mathrm{i}}$ and $a_{\mathrm{i}}=A_{\mathrm{i}} / \Delta V$ are the interfacial convective heat transfer coefficient and surface area per unit volume, respectively. The terms describing the convective transport in Eq. (1.32) have the following physical significance (see Rocamora and de Lemos (2000) [23] for details): I, macroscopic convective transport; II, thermal dispersion associated with deviations of time-averaged local velocity and temperature (note that this term also appears when analyzing laminar convection in porous media); III, turbulent heat flux due to the fluctuating components of macroscopic velocity and temperature; and IV, turbulent thermal dispersion in a porous medium due to both time fluctuations and spatial deviations of both microscopic velocity and temperature.

\subsection{4}

\section{Macroscopic Two-Energy Equation Modeling}

In order to apply Eqs. (1.32) and (1.33) to obtain the temperature fields for turbulent flow in porous media, unknown terms in Eq. (1.32) have to be modeled in some way as a function of the intrinsically averaged temperatures of solid and fluid phases, $\left\langle\overline{T_{\mathrm{s}}}{ }^{\mathrm{i}}\right.$ and $\left\langle\overline{T_{\mathrm{f}}}\right\rangle^{\mathrm{i}}$, respectively. To accomplish this, a gradient-type diffusion model is used for all unknown terms, i.e. thermal dispersion due to spatial deviations, turbulent heat flux due to temporal fluctuations and turbulent thermal dispersion due to both temporal fluctuations and spatial deviations. Also needed is a model for local conduction. Using these gradient-type diffusion models, we can write

$$
\begin{aligned}
& \text { Thermal dispersion: }-\left(\rho c_{\mathrm{p}}\right)_{\mathrm{f}}\left(\phi\left\langle\overline{\mathbf{u}}^{i} \overline{T_{\mathrm{f}}}\right\rangle^{\mathrm{i}}\right)=\mathbf{K}_{\mathrm{disp}} \cdot \nabla\left\langle\overline{T_{\mathrm{f}}}\right\rangle^{\mathrm{i}} \\
& \text { Turbulent heat flux: }-\left(\rho c_{\mathrm{p}}\right)_{\mathrm{f}}\left(\phi \overline{\left\langle\mathbf{u}^{\prime}\right\rangle^{i}\left\langle T_{\mathrm{f}}^{\prime}\right\rangle^{i}}\right)=\mathbf{K}_{\mathrm{t}} \cdot \nabla\left\langle\bar{T}_{\mathrm{f}}\right\rangle^{\mathrm{i}} \\
& \text { Turbulent thermal dispersion: } \left.-\left(\rho c_{\mathrm{p}}\right)_{\mathrm{f}}\left(\boldsymbol{\phi} \overline{\left\langle^{\overline{\mathbf{u}^{\prime}}} \bar{T}_{\mathrm{f}}^{\prime}\right.}\right\rangle^{\mathrm{i}}\right)=\mathbf{K}_{\text {disp,t }} \cdot \nabla\left\langle\bar{T}_{\mathrm{f}}\right\rangle^{\mathrm{i}}
\end{aligned}
$$


Local conduction: $\left\{\begin{array}{l}\frac{1}{\Delta V} \int_{A_{i}} \mathbf{n}_{\mathrm{i}} k_{\mathrm{f}} \overline{T_{\mathrm{f}}} \mathrm{d} A=\mathbf{K}_{\mathrm{f}, \mathrm{s}} \cdot \nabla\left\langle\overline{T_{\mathrm{s}}}\right\rangle^{\mathrm{i}} \\ \frac{1}{\Delta V} \int_{A_{i}} \mathbf{n}_{\mathrm{i}} k_{\mathrm{s}} \overline{T_{\mathrm{s}}} \mathrm{d} A=\mathbf{K}_{\mathrm{s}, \mathrm{f}} \cdot \nabla\left\langle\overline{T_{\mathrm{f}}}\right\rangle^{\mathrm{i}}\end{array}\right.$

For the above expressions, Eqs. (1.32) and (1.33) can be written as

$$
\begin{aligned}
& \left\{\left(\rho c_{\mathrm{p}}\right)_{\mathrm{f}} \phi\right\} \frac{\partial\langle\bar{T}\rangle^{\mathrm{i}}}{\partial t}+\left(\rho c_{\mathrm{p}}\right)_{\mathrm{f}} \nabla \cdot\left(\mathbf{u}_{\mathrm{D}}\left\langle\overline{T_{\mathrm{f}}}\right\rangle^{\mathrm{i}}\right)=\nabla \cdot\left\{\mathbf{K}_{\mathrm{eff}, \mathrm{f}} \cdot \nabla\left\langle\overline{T_{\mathrm{f}}}\right\rangle^{\mathrm{i}}\right\}+h_{\mathrm{i}} a_{\mathrm{i}}\left(\left\langle\overline{T_{\mathrm{s}}}\right\rangle^{\mathrm{i}}-\left\langle\overline{T_{\mathrm{f}}}\right\rangle^{\mathrm{i}}\right) \\
& \left\{(1-\phi)\left(\rho c_{\mathrm{p}}\right)_{\mathrm{s}}\right\} \frac{\partial\langle\bar{T}\rangle^{\mathrm{i}}}{\partial t}=\nabla \cdot\left\{\mathbf{K}_{\mathrm{eff}, \mathrm{s}} \cdot \nabla\left\langle\overline{T_{\mathrm{s}}}\right\rangle^{\mathrm{i}}\right\}+h_{\mathrm{i}} a_{\mathrm{i}}\left(\left\langle\overline{T_{\mathrm{f}}}\right\rangle^{\mathrm{i}}-\left\langle\overline{T_{\mathrm{s}}}\right\rangle^{\mathrm{i}}\right)
\end{aligned}
$$

where $\mathbf{K}_{\mathrm{eff,f}}$ and $\mathbf{K}_{\mathrm{eff}, \mathrm{s}}$ are the effective conductivity tensors for the fluid and solid phases, respectively, given by

$$
\begin{aligned}
& \mathbf{K}_{\mathrm{eff}, \mathrm{f}}=\left[\phi k_{\mathrm{f}}\right] \mathbf{I}+\mathbf{K}_{\mathrm{f}, \mathrm{s}}+\mathbf{K}_{\mathrm{disp}}+\mathbf{K}_{\mathrm{disp}, \mathrm{t}}+\mathbf{K}_{\mathrm{t}} \\
& \mathbf{K}_{\mathrm{eff}, \mathrm{s}}=\left[(1-\phi) k_{\mathrm{s}}\right] \mathbf{I}+\mathbf{K}_{\mathrm{s}, \mathrm{f}}
\end{aligned}
$$

and $\mathbf{I}$ is the unit tensor.

Further, in order to be able to apply Eq. (1.38), it is necessary to determine the components of the conductivity tensor in Eq. (1.40), i.e. $\mathbf{K}_{\mathrm{f}, \mathrm{s}}, \mathbf{K}_{\mathrm{disp}}, \mathbf{K}_{\mathrm{t}}$ and $\mathbf{K}_{\mathrm{disp}, \mathrm{t}}$. Following Kuwahara and Nakayama (1996) [42] and Quintard et al. (1997) [10], this can be accomplished for the thermal dispersion and local conduction tensors, $\mathbf{K}_{\text {disp }}$ and $\mathbf{K}_{\mathrm{f}, \mathrm{s}}$, by making use of a unit cell subjected to periodic boundary conditions for the flow together with an imposed linear temperature gradient on the porous medium. The dispersion and conduction tensors are then obtained directly from the distributed results within the unit cell by making use of Eqs. (1.34) and (1.37). In addition, the following correlations by Nakayama and Kuwahara (1999) [46] for the thermal dispersion tensor, which are valid for $P e_{\mathrm{D}} \geq 10$, can be used:

$$
\begin{aligned}
& \frac{\left(K_{\text {disp }}\right)_{x x}}{k_{\mathrm{f}}}=2.1 \frac{P e_{\mathrm{D}}}{(1-\phi)^{0.1}} \quad \text { for longitudinal dispersion } \\
& \frac{\left(K_{\mathrm{disp}}\right)_{Y Y}}{k_{\mathrm{f}}}=0.052(1-\phi)^{0.5} P e_{\mathrm{D}} \quad \text { for transverse dispersion }
\end{aligned}
$$

where $\left(K_{\text {disp }}\right)_{x x}$ and $\left(K_{\text {disp }}\right)_{Y y}$ are the longitudinal and transverse components of $\mathbf{K}_{\text {disp }}$, respectively.

The turbulent heat flux and turbulent thermal dispersion components of $\mathbf{K}_{\mathrm{eff}, \mathrm{f}}$, namely $\mathbf{K}_{\mathrm{t}}$ and $\mathbf{K}_{\mathrm{disp}, \mathrm{t}}$, respectively, are not determined from a distributed calculation. Instead, they are modeled through the classical eddy diffusivity concept, 
similar to Nakayama and Kuwahara (1999) [46]. It should be noticed that these two terms arise only if the flow is turbulent within the void space, whereas the thermal dispersion term, $\mathbf{K}_{\text {disp }}$, exists for both laminar and turbulent flow regimes. Starting out from the time-averaged local energy equation coupled with the standard modeling of the turbulent heat flux through the eddy diffusivity concept, $v_{\mathrm{t}}$, one can write

$$
-\left(\rho c_{\mathrm{p}}\right)_{\mathrm{f}} \overline{\mathbf{u}^{\prime} T_{\mathrm{f}}^{\prime}}=\left(\rho c_{\mathrm{p}}\right)_{\mathrm{f}} \frac{\nu_{\mathrm{t}}}{\sigma_{\mathrm{t}}} \nabla \bar{T}_{\mathrm{f}}
$$

where $\sigma_{\mathrm{t}}$ is the turbulent Prandtl number, which is taken here as a constant.

Applying the volume average to the resulting equation, one obtains the macroscopic version of the turbulent heat flux, given by

$$
-\left(\rho c_{\mathrm{p}}\right)_{\mathrm{f}} \phi\left\langle\overline{\mathbf{u}^{\prime} T_{\mathrm{f}}^{\prime}}\right\rangle^{\mathrm{i}}=\left(\rho c_{\mathrm{p}}\right)_{\mathrm{f}} \frac{\nu_{\mathrm{t}_{\mathrm{f}}}}{\sigma_{\mathrm{t}}} \nabla\left\langle\bar{T}_{\mathrm{f}}\right\rangle^{\mathrm{i}}
$$

where we have adopted the symbol $v_{t_{\phi}}$ to express the macroscopic eddy diffusivity. Now, adding up Eqs. (1.35) and (1.36) in light of Eq. (1.30) one has

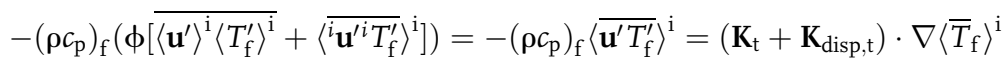

According to Eqs. (1.45) and (1.46), the overall turbulent heat transport is the sum of the turbulent heat flux and the turbulent thermal dispersion mechanisms, as proposed by Rocamora and de Lemos (2000) [23]. As suggested by Eq. (1.45), both mechanisms are modeled together, giving for $\mathbf{K}_{\mathrm{t}}$ and $\mathbf{K}_{\mathrm{disp}, \mathrm{t}}$ the expression

$$
\mathbf{K}_{\mathrm{t}}+\mathbf{K}_{\mathrm{disp}, \mathrm{t}}=\phi\left(\rho c_{\mathrm{p}}\right)_{\mathrm{f}} \frac{\nu_{\mathrm{t}_{\phi}}}{\sigma_{\mathrm{t}}} \mathbf{I}
$$

Details of interfacial convective heat transfer coefficient are presented in the next section.

\section{2 .5}

\section{Interfacial Heat Transfer Coefficient}

In Eqs. (1.32) and (1.33) the heat transferred between the two phases was modeled by means of a film coefficient $h_{\mathrm{i}}$ such that

$$
h_{\mathrm{i}} a_{\mathrm{i}}\left(\left\langle\overline{T_{\mathrm{s}}}\right\rangle^{\mathrm{i}}-\left\langle\overline{T_{\mathrm{f}}}\right\rangle^{\mathrm{i}}\right)=\frac{1}{\Delta V} \int_{A_{i}} \mathbf{n}_{\mathrm{i}} \cdot k_{\mathrm{f}} \nabla \overline{T_{\mathrm{f}}} \mathrm{d} A=\frac{1}{\Delta V} \int_{A_{i}} \mathbf{n}_{\mathrm{i}} \cdot k_{\mathrm{s}} \nabla \overline{T_{\mathrm{s}}} \mathrm{d} A
$$

where $a_{\mathrm{i}}$, as mentioned, is the interfacial area per unit volume. In foam-like or cellular media, the high values of $a_{\mathrm{i}}$ make them attractive for transferring thermal energy via conduction through the solid followed by convection to a fluid stream. 
a)
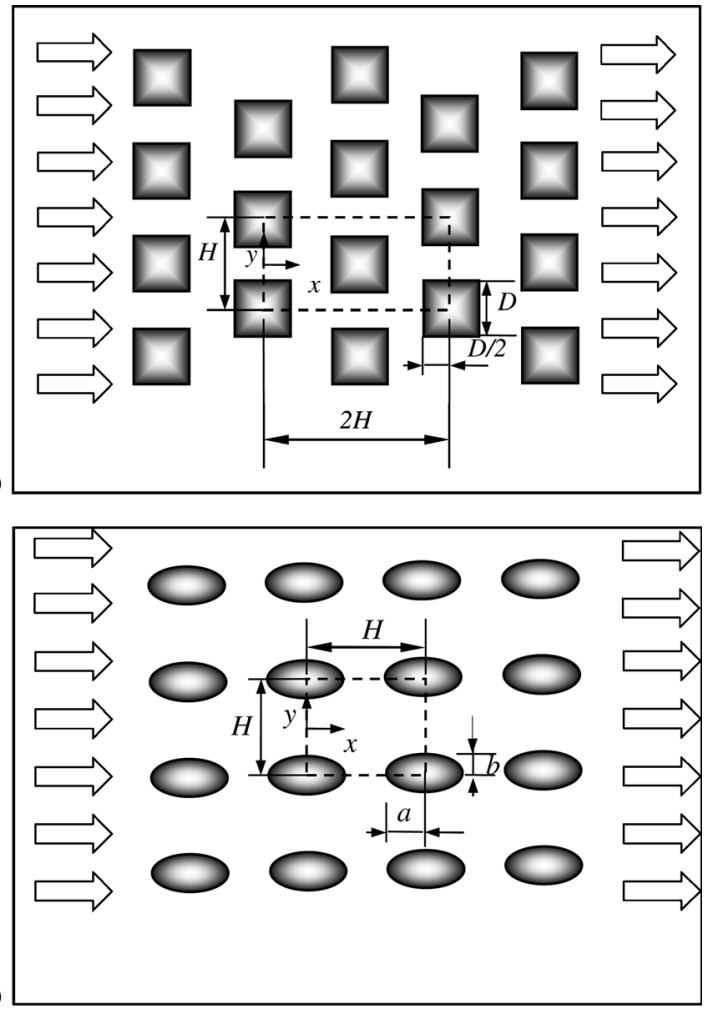

Figure 1.1 Porous media models and coordinate systems:

(a) triangular (staggered) array of square rods; (b) square (inline) array of elliptic rods.

For obtaining macroscopic transport properties, highly permeable media can be modeled as an infinite array of rods, which, in turn, can be analogous to flow across a bundle of tubes. Accordingly, two tube arrangements are generally found in the literature, i.e. the tube rows in a bundle are either inline, with rod centers forming a square or a rectangle, or else they are staggered, where a triangular shape is obtained when connecting the tube centerlines. In this chapter, the two forms of arrays, namely square (inline) and triangular (staggered) layouts are used in order to model flow and heat transfer in highly porous media (see Figure 1.1).

For the staggered configuration of tube banks, Zhukauskas (1972) [56] has proposed a correlation of the form

$$
\frac{h_{\mathrm{i}} D}{k_{\mathrm{f}}}=0.022 R e_{\mathrm{D}}^{0.84} \operatorname{Pr}^{0.36} \quad \text { for } 2 \times 10^{5}<R e_{\mathrm{D}}<2 \times 10^{6}
$$

where the values 0.022 and 0.84 are for tubes in cross flow. 
Wakao et al. (1979) [57] obtained a heuristic correlation for a closely packed bed of particle diameter $D$ and compared their results with experimental data. This correlation for the interfacial heat transfer coefficient is given by

$$
\frac{h_{\mathrm{i}} D}{k_{\mathrm{f}}}=2+1.1 \operatorname{Re}_{\mathrm{D}}^{0.6} \operatorname{Pr}^{1 / 3}
$$

For numerically determining $h_{\mathrm{i}}$, Kuwahara et al. (2001) [12] modeled a porous medium by considering it as an infinite number of solid square rods of size $D$, arranged in a regular triangular pattern (see Figure 1.1). They numerically solved the governing equations in the void region, exploiting to advantage the fact that for an infinite and geometrically ordered medium a repetitive cell can be identified. Periodic boundary conditions were then applied for obtaining the temperature distribution under fully developed flow conditions. A numerical correlation for the interfacial convective heat transfer coefficient was proposed by Kuwahara et al. (2001) [12] for laminar flow as

$$
\frac{h_{\mathrm{i}} D}{k_{\mathrm{f}}}=\left(1+\frac{4(1-\phi)}{\phi}\right)+\frac{1}{2}(1-\phi)^{1 / 2} \operatorname{Re}_{\mathrm{D}} \operatorname{Pr}^{1 / 3}, \quad \text { valid for } 0.2<\phi<0.9
$$

Equation (1.51) is based on porosity dependency and is valid for packed beds of particle diameter D. Saito and de Lemos (2005) [15] obtained the interfacial heat transfer coefficient for laminar flows though an infinite square rod using the same methodology as Kuwahara et al. (2001) [12].

\section{3}

\section{Numerical Determination of $h_{\mathrm{i}}$}

\subsection{1}

\section{Physical Model}

Measuring flow and heat transfer characteristics within the void space in foam-like media is a challenging task. However, macroscopic behavior of permeable materials can be obtained by integrating distributed parameters calculated at pore scale. In order to follow such methodology, scientists and engineers have made use of physical models that consider a well-ordered porous medium, which is composed by regularly arranged obstacled instead of randomly distributed solid particles. Assuming further that such medium is of a large size, a repetitive or unit all can be identified, over which the balance equations are then numerically solved.

Following this path, Kuwahara et al. (2001) [12] and Nakayama et al. (2001) [13] modeled a porous medium in terms of square obstacles displaced in a regular staggered pattern. They numerically solved the set of local governing equations in a unit or repetitive cell of that arrangement. By volume averaging the distributed 
a)

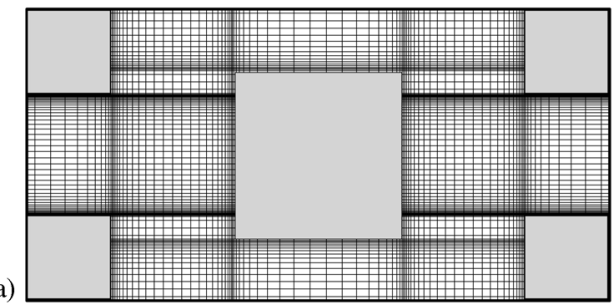

b)

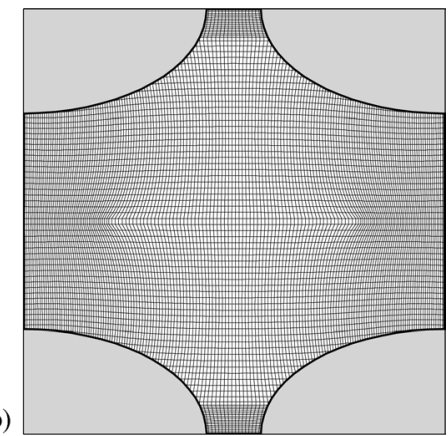

Figure 1.2 Periodic cells and computational grids: (a) triangular (staggered) array of square rods; (b) square (aligned) array of elliptic rods.

parameters, they got useful information used in calculating the interfacial heat transfer coefficient $h_{\mathrm{i}}$. Such coefficient, as seen above, is necessary to close the mathematical model when two energy equations, one for the solid and one for the saturating fluid, are solved.

Motivated by the foregoing, this work also applies the methodology of computing first distributed flow parameters in a repetitive cell followed by integration of local values. The periodic cell of volume $\Delta V$ used in this work is schematically shown in Figure 1.1. It has dimensions $2 H \times H$ for square rods (Figure 1.1a) and $H \times H$ for elliptic obstacles (Figure 1.1b). Computations within those cells were carried out using a nonuniform grid as presented in Figure 1.2. The Reynolds number $R e_{\mathrm{D}}=\rho \overline{\mathbf{u}}_{\mathrm{D}} D / \mu$ was varied from $10^{4}$ to $10^{7}$.

The numerical method utilized to discretize the flow and energy equations in the unit cell was the finite control volume approach. The SIMPLE method of Patankar [58] was used for handling the pressure-velocity coupling. Convergence was monitored in terms of the normalized residue for all variables. The maximum residue allowed before convergence was $10^{-9}$, being the variables normalized by appropriate reference values.

For fully developed flow in the cells of Figure 1.1, the velocity at exit $(x / H=2$ for square rods and $x / H=1$ for elliptic rods) must be identical to that at the inlet $(x / H=0)$. Temperature profiles, however, are only identical at both cell exit and inlet if presented in terms of an appropriate nondimensional variable. The situation is analogous to the case of forced convection in a channel with isothermal walls. Due to the periodicity of the flow, a single structural unit, as indicated in Figure 1.1, may be taken as a calculation domain. Turbulent flow is modeled by means of Eqs. (1.25), (1.26) and (1.27). Boundary conditions are given by the following.

(a) On the solid walls (laminar or low Re model):

$$
\bar{u}=0, k=0, \varepsilon=v \frac{\partial^{2} k}{\partial y^{2}}, \bar{T}=\bar{T}_{\mathrm{w}}
$$


(b) On the solid walls (high Re model):

$$
\frac{\bar{u}}{u_{\tau}}=\frac{1}{\kappa} \ln \left(\gamma^{+} E\right), k=k_{w}=\frac{u_{\tau}^{2}}{c_{\mu}^{1 / 2}}, \varepsilon=\frac{c_{\mu}^{3 / 4} k_{\mathrm{w}}^{3 / 2}}{\kappa \gamma_{\mathrm{w}}}, q_{\mathrm{w}}=\frac{\left(\rho c_{\mathrm{p}}\right)_{\mathrm{f}} c_{\mu}^{1 / 4} k_{\mathrm{w}}^{1 / 2}\left(\bar{T}-T_{\mathrm{w}}\right)}{\left(\frac{\sigma_{t}}{\kappa} \ln \left(\gamma_{\mathrm{w}}^{+}\right)+c_{\mathrm{Q}}(\operatorname{Pr})\right)}
$$

where $u_{\tau}=\left(\tau_{\mathrm{w}} / \rho\right)^{1 / 2}, \gamma_{\mathrm{w}}^{+}=\gamma_{\mathrm{w}} u_{\tau} / \nu, c_{\mathrm{Q}}=12.5 \operatorname{Pr}^{2 / 3}+2.12 \ln (\operatorname{Pr})-5.3$ for $\operatorname{Pr}>0.5$.

In Eq. (1.53), $P r$ and $\sigma_{\mathrm{t}}$ are the Prandtl and turbulent Prandtl numbers, respectively, $q_{\mathrm{w}}$ is wall heat flux, $u_{\tau}$ is wall-friction velocity, $\gamma_{\mathrm{w}}$ is the normal coordinate of the first grid point close to the wall and $\kappa$ is the von Kármán constant. Further, in Eq. (1.53) $E$ is also a constant, which can accommodate different types of surface. For smooth surfaces, a standard numerical value taken for $E$ is 9.0. The other boundary conditions are the following.

(c) On the symmetry planes:

$$
\frac{\partial \bar{u}}{\partial y}=\frac{\partial k}{\partial y}=\frac{\partial \varepsilon}{\partial y}=0
$$

(d) On the periodic boundaries:

$$
\begin{aligned}
& \left.\bar{u}\right|_{\text {inlet }}=\left.\bar{u}\right|_{\text {outlet }},\left.\bar{v}\right|_{\text {inlet }}=\left.\bar{v}\right|_{\text {outlet }},\left.k\right|_{\text {inlet }}=\left.k\right|_{\text {outlet }},\left.\varepsilon\right|_{\text {inlet }}=\left.\varepsilon\right|_{\text {outlet }} \text {, } \\
& \left.\theta\right|_{\text {inlet }}=\left.\left.\theta\right|_{\text {outlet }} \Leftrightarrow \frac{\bar{T}-\bar{T}_{\mathrm{w}}}{\bar{T}_{\mathrm{B}}(x)-\bar{T}_{\mathrm{w}}}\right|_{\text {inlet }}=\left.\frac{\bar{T}-\bar{T}_{\mathrm{w}}}{\bar{T}_{\mathrm{B}}(x)-\bar{T}_{\mathrm{w}}}\right|_{\text {outlet }}
\end{aligned}
$$

The bulk mean temperature of the fluid is given by

$$
\bar{T}_{\mathrm{B}}(x)=\frac{\int \bar{u} \bar{T} \mathrm{~d} y}{\int \bar{u} \mathrm{~d} y}
$$

Computations are based on the Darcy velocity, the length of structural unit $H$ and the temperature difference $\left(\bar{T}_{\mathrm{B}}(x)-\bar{T}_{\mathrm{w}}\right)$ as reference scales.

\subsection{2}

\section{Periodic Flow}

Results for velocity and temperature fields were obtained for different Reynolds numbers. In order to ensure that the flow was hydrodynamically and thermally developed in the periodic cell of Figure 1.1, the governing equations were solved repetitively in the cell, taking the outlet profiles for $\overline{\mathbf{u}}$ and $\theta$ at the exit and plugging them back at the inlet. In the first run, uniform velocity and temperature profiles were set at the cell entrance for $\operatorname{Pr}=1$ giving $\theta=1$ at $x / H=0$. Then, after convergence of the flow and temperature fields, $\overline{\mathbf{u}}$ and $\theta$ at $x / H=2$ were used as inlet profiles for a second run, corresponding to solving again the flow for a similar cell beginning in $x / H=2$. Similarly, a third run was carried out and again outlet results, 
this time corresponding to an axial position $x / H=4$, were recorded. This procedure was repeated several times until $\overline{\mathbf{u}}$ and $\theta$ did not differ substantially at both inlet and outlet positions, as obtained in Saito and de Lemos (2006) [38].

For the low Re model, the first node adjacent to the wall requires that the nondimensional wall distance be such that $\gamma^{+}=u_{\tau} \gamma \rho / \mu \leq 1$. To accomplish this requirement, the grid needs a great number of points close to the wall leading to computational meshes of large sizes.

\subsection{3}

\section{Film Coefficient $h_{\mathrm{i}}$}

The determination of $h_{\mathrm{i}}$ is here obtained by calculating, for the unit cell of Figure 1.1, an expression given as

$$
h_{\mathrm{i}}=\frac{Q_{\mathrm{total}}}{A_{\mathrm{i}} \Delta T_{\mathrm{ml}}}
$$

where the overall heat transferred in the cell, $Q_{\text {total }}$, is given by

$$
Q_{\text {total }}=A_{\mathrm{c}} \rho \bar{u}_{\mathrm{B}} c_{\mathrm{p}}\left(\left.\bar{T}_{\mathrm{B}}\right|_{\text {outlet }}-\left.\bar{T}_{\mathrm{B}}\right|_{\text {inlet }}\right)
$$

and $A_{\mathrm{i}}$ and $A_{\mathrm{c}}$ are presented in Table 1.1. The bulk mean velocity of the fluid is given by

$$
\bar{u}_{\mathrm{B}}(x)=\frac{\int \bar{u} \mathrm{~d} y}{\int \mathrm{d} y}
$$

and the logarithm mean temperature difference, $\Delta T_{\mathrm{ml}}$, is defined as

$$
\Delta T_{\mathrm{ml}}=\frac{\left(\bar{T}_{\mathrm{w}}-\left.\bar{T}_{\mathrm{B}}\right|_{\text {outlet }}\right)-\left(\bar{T}_{\mathrm{w}}-\left.\bar{T}_{\mathrm{B}}\right|_{\text {inlet }}\right)}{\ln \left[\left(\bar{T}_{\mathrm{w}}-\left.\bar{T}_{\mathrm{B}}\right|_{\text {outlet }}\right)\left(\bar{T}_{\mathrm{w}}-\left.\bar{T}_{\mathrm{B}}\right|_{\text {inlet }}\right)\right]}
$$

Equation (1.59) represents an overall heat balance on the entire cell and Eq. (1.58) associates the heat transferred to the fluid with a suitable temperature difference. As mentioned earlier, Eqs. (1.1) to (1.4) were solved numerically in the unit cell subjected to conditions (1.55) and (1.56). Once fully developed flow and nondimensional temperature fields were achieved, bulk temperatures were calculated according to Eq. (1.57), at both inlet and outlet positions. They were then used to calculate $h_{\mathrm{i}} D / k_{\mathrm{f}}$ using Eqs. (1.58) to (1.61).

Table 1.1 Interface $\left(A_{i}\right)$ and flow $\left(A_{c}\right)$ areas for distinct rod arrangements.

\begin{tabular}{lll}
\hline Geometry & $\boldsymbol{A}_{\mathbf{i}}$ & $\boldsymbol{A}_{\mathbf{c}}$ \\
\hline Square rods - triangular array & $8 D$ & $H-D$ \\
Elliptic rods - square array & $2 \pi \sqrt{0.5\left(a^{2}+b^{2}\right)}$ & $H-2 b$
\end{tabular}


1.4

Results and Discussion

1.4. 1

Array of Square Rods

Figure 1.3 shows distributed temperature fields for distinct cell porosities. One can see that the lower the porosity (Figure 1.3a), the higher the average fluid temperatures are for the same mass flow through the bed (same $R e_{\mathrm{D}}$ ). This is an indication that the transfer of heat from the solid to the fluid phase is enhanced leading to higher value for $h_{\mathrm{i}}$ as $\phi$ is reduced. Corresponding results for the heat transfer coefficient are plotted in Figure 1.4 for $R e_{\mathrm{D}}$ up to 400 . Also plotted in this figure are results computed with Kuwahara et al. (2001) [12] correlation, Eq. (1.51), using different porosity values. The figure clearly indicates that both computations show a reasonable agreement. As mentioned, it is also clearly seen from Figure 1.4 that the lower the porosity, the higher the value of the Nusselt number. Increasing of $h_{\mathrm{i}}$ with $R e_{\mathrm{D}}$ is also observed.

Macroscopically developed flow field for $\operatorname{Pr}=1$ and $R e_{\mathrm{D}}=10^{5}$ are presented in Figure 1.5, corresponding to the axial position $x / D=6$ along the longitudinal coordinate (see Figure 1.1). The expression "macroscopically developed" is used herein to account for the fact that periodic flow has been achieved at that axial position. The figure shows the velocity field, streamlines, pressure and turbulent kinetic energy distributions for $\phi=0.65$. The flow impinges on the left face of the obstacles (Figure 1.5a), surrounds the rod faces and forms a weak recirculation bubble past the rod (Figure 1.5b). Pressure increases at the front face of the rod and drastically decreases behind the obstacles, as can be seen from the pressure contours shown in Figure 1.5c. Figure 1.5d illustrates levels of turbulence kinetic energy, which are higher around the rod corners where a strong shear layer is formed. Further downstream of the rods, in the wake region, steep velocity gradients appear due to flow deceleration, increasing there also the local level of turbulence kinetic energy.

Corresponding temperature distribution is shown in Figure 1.6, also for $R e_{\mathrm{D}}=10^{5}$ and $\phi=0.65$. Colder fluid impinges on the rod left side yielding strong temperature gradients on that face. Downstream of the obstacles, fluid recirculation smoothes temperature gradients and deforms isotherms within the mixing region, which is here more clearly seen with a staggered geometry. When the Reynolds number is sufficiently high, thermal boundary layers cover the rod surfaces indicating that convective heat transfer overwhelms thermal diffusion.

\section{4 .2}

\section{Array of Elliptic Rods}

Figure 1.7 presents the temperature fields corresponding to different $R e_{\mathrm{D}}$ and porosities, covering the laminar flow range $100 \leq R e_{\mathrm{D}} \leq 1000$. Inspecting the figure, one can observe that the lower the porosity, the more efficiently heat is added to the fluid. Consequently, values of $h_{\mathrm{i}}$ tend to be higher for lower values of $\phi$, in a fashion 
1.4 Results and Discussion $\mid 17$

a)
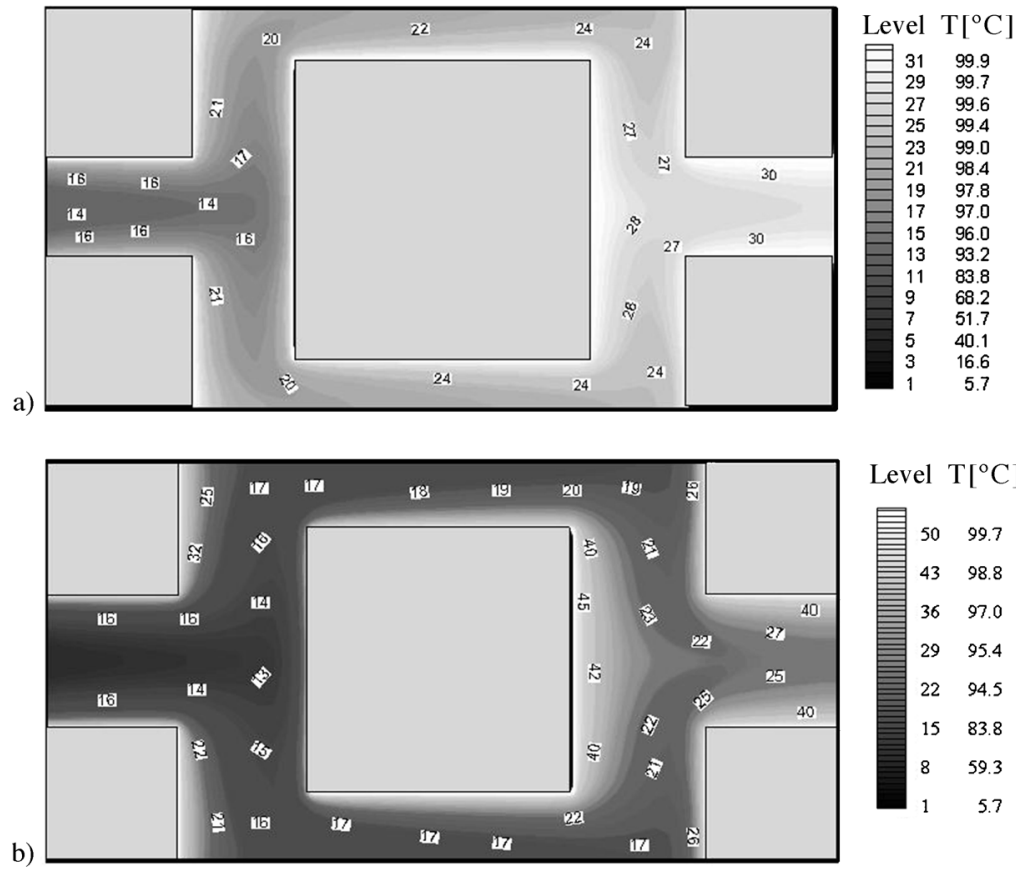

Level $\mathrm{T}\left[{ }^{\circ} \mathrm{C}\right]$
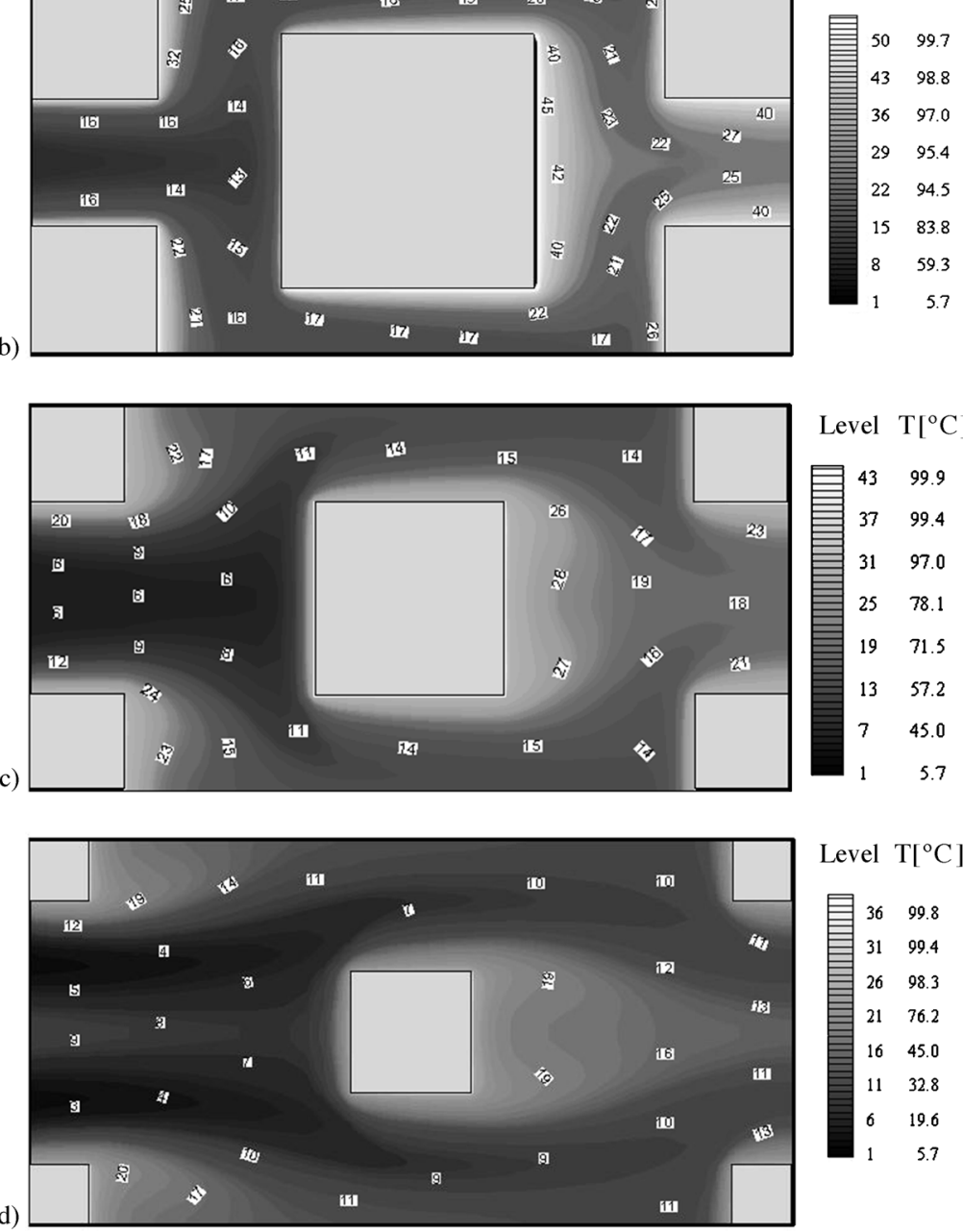

Figure 1.3 Temperature fields in a periodic cell for square rods and $R e_{\mathrm{D}}=100$;

(a) $\phi=0.44$; (b) $\phi=0.55$; (c) $\phi=0.75$; (d) $\phi=0.90$. 


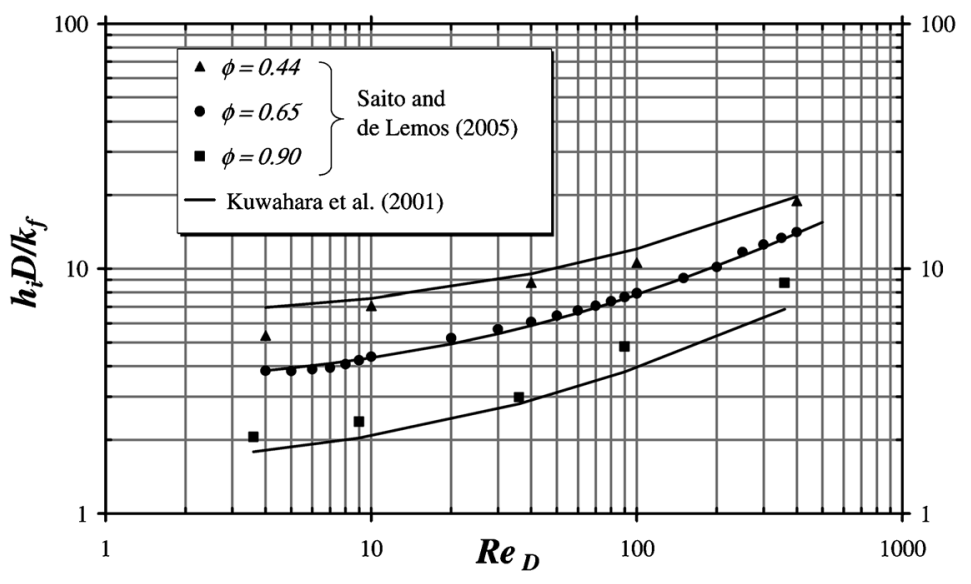

Figure 1.4 Effect of $R e_{\mathrm{D}}$ and $\phi$ on $h_{\mathrm{i}}$ and comparison with correlation of Kuwahara et al. [12] (data from [15]).

similar to what occurred with the square rod case seen above. Also, as $R e_{\mathrm{D}}$ increases, heat is transferred more effectively to the flow, as expected. Figure 1.8 compares integrated $h_{\mathrm{i}}$ values for square and elliptic rods for porosities $\phi=0.75$ and $\phi=0.90$. Also shown are results by Kuwahara et al. (2001) [12]. It is interesting to note that the more streamlined flow over elliptic rods, which are displaced in an aligned manner, is less effective in promoting heat transfer between phases. Also, the higher the $R e_{\mathrm{D}}$ number, the higher the heat transfer coefficient. Figure 1.9 compiles $h_{\mathrm{i}}$ values as a function of porosity and $R e_{\mathrm{D}}$ up to 1000 . As in the case of square obstacles, the lower the porosity the higher the heat transfer rate for the same mass flux across the bed. However, such dependency on $\phi$ appears to be weaker than in the cases of square rods, if one compares the wider spread of data as porosity varies in Figure 1.4. In addition, as $R e_{\mathrm{D}}$ increases, the effect of $\phi$ on $h_{\mathrm{i}}$ becomes less pronounced. Also, the figure seems to indicate two distinct regimes, the first one for $R e_{\mathrm{D}}<100$ and a second for $R e_{\mathrm{D}}>100$. However, the limited amount of data presented here does not allow more definite conclusions to be drawn.

Results for high Re numbers are considered next. Figure 1.10 shows distributions of pressure, turbulence kinetic energy and temperature fields in the periodic cell of Figure $1.2 \mathrm{~b}$, which were obtained at $R e_{\mathrm{D}}=10^{5}$ for the cases of $\phi=0.60$ and $\phi=0.90$. One can observe that pressure increases at the left face of the rods and decreases behind them, with the surface peak pressure moving towards the stagnation point on the left, along the ellipse horizontal axis, as $\phi$ increases. The turbulence kinetic energy distribution is presented in Figures $1.10 \mathrm{c}$ and $\mathrm{d}$. Levels of $k$ are higher close to the walls and along the converging section of the channel where a strong shear layer prevails. The temperature distribution pattern is presented in Figures 1.10e and $\mathrm{f}$ showing that a thermal boundary layer covers most of the surface, possibly indicating that convective heat transfer overwhelms thermal diffusion for the case of high mass flow rates. 
a)

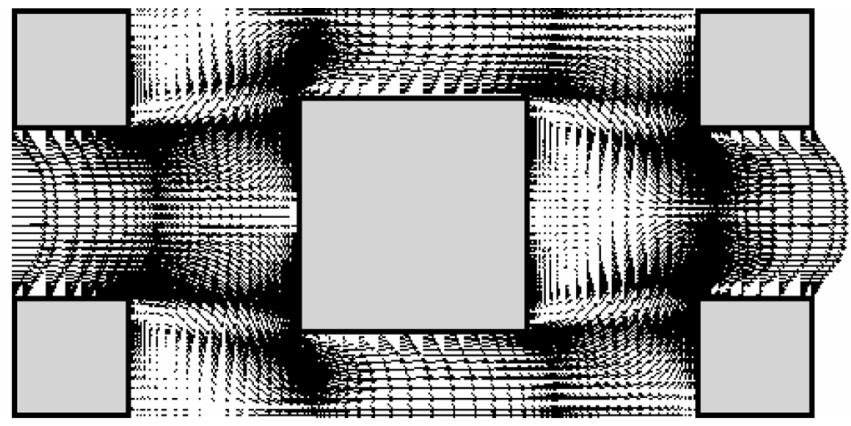

b)

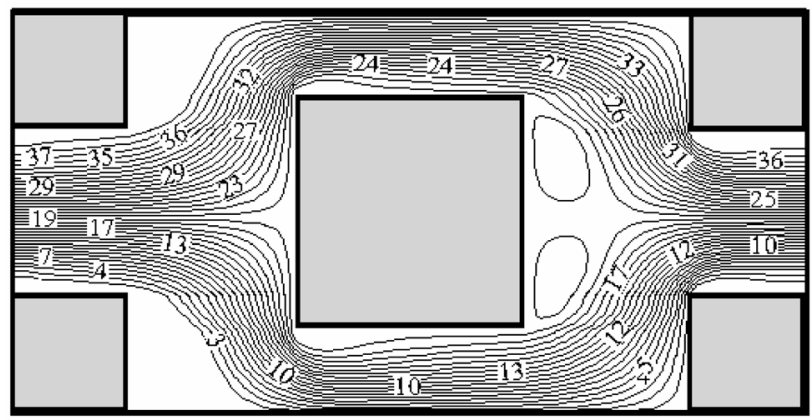

Level Stream

$$
\begin{array}{ll}
36 & 2.2 \mathrm{E}-01 \\
31 & 1.9 \mathrm{E}-01 \\
26 & 1.6 \mathrm{E}-01 \\
21 & 1.3 \mathrm{E}-01 \\
16 & 9.9 \mathrm{E}-02 \\
11 & 6.8 \mathrm{E}-02 \\
6 & 3.7 \mathrm{E}-02 \\
1 & 5.4 \mathrm{E}-03
\end{array}
$$

c)

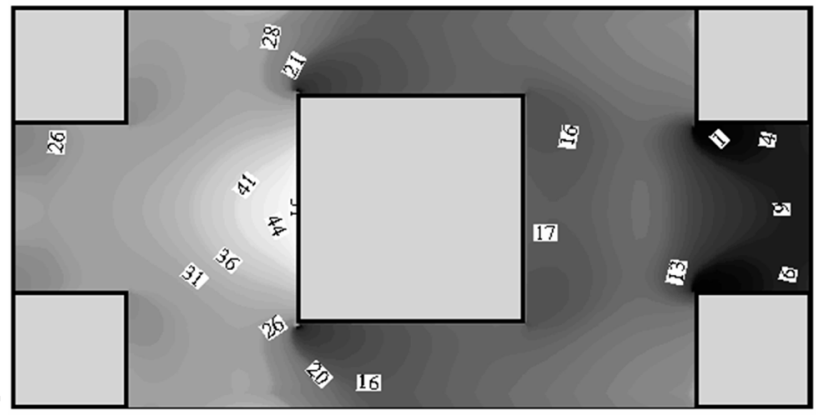

Level $\mathrm{P}^{*}$
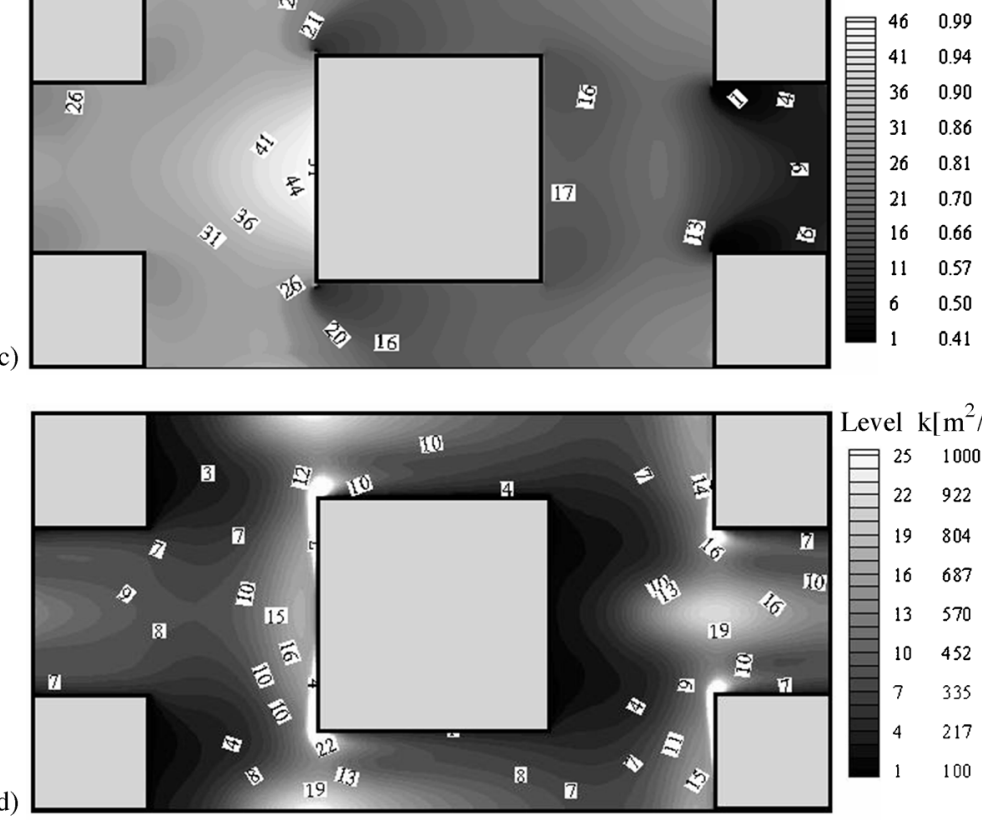

Level $\mathrm{k}\left[\mathrm{m}^{2} / \mathrm{s}^{2}\right]$

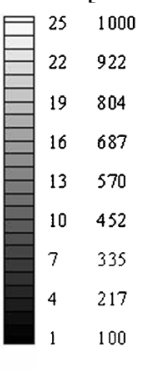

Figure 1.5 Spatially periodic flow and pressure fields, $R e_{\mathrm{D}}=10^{5}$ and $\phi=0.65$;

(a) vector plot; (b) streamlines; (c) pressure; (d) turbulent kinetic energy. 
$20 \mid 1$ Interfacial Heat Transport in Highly Permeable Media

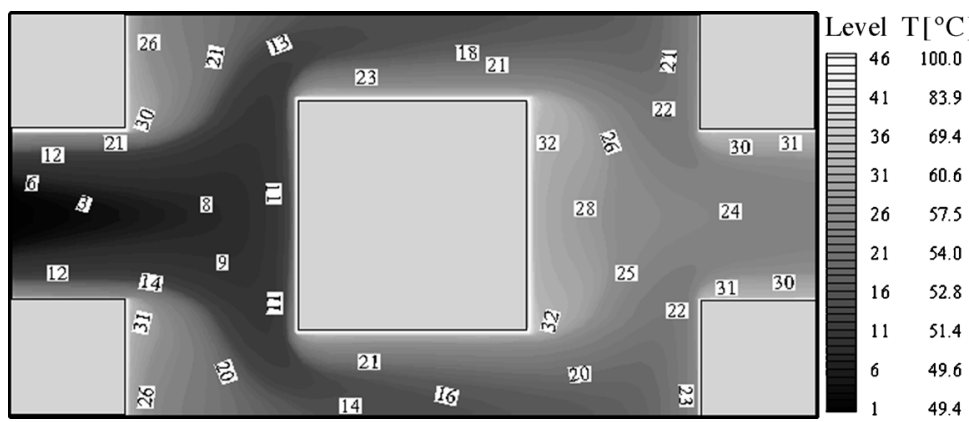

Figure 1.6 Spatially periodic temperature field, $R e_{\mathrm{D}}=10^{5}$ and $\phi=0.65$.

Results for $h_{\mathrm{i}} D / k_{\mathrm{f}}$ are plotted in Figure 1.11 for $R e_{\mathrm{D}}$ up to $10^{7}$. Also plotted in this figure are results computed with correlation 1.51 by Kuwahara et al. (2001) [12] using $\phi=0.65$. The figure seems to indicate that computations show a reasonable agreement for laminar results. In addition, numerical results for turbulent flow using low and high Re models are also presented in this figure. Differences in the flow pattern between the cases here reported, namely aligned-elliptic rods and staggered-square obstacles, are reflected in the lower heat transfer coefficients for the former case. Sharpe edges of square obstacles, disposed in a triangular arrangement, agitate the fluid in a much stronger fashion that in the case of well-behaved streamlined flow across elliptic tubes.

Figure 1.12 shows numerical results for the interfacial convective heat transfer coefficient for porosities $\phi=0.65$ and $\phi=0.90$. Results for $h_{\mathrm{i}} D / k_{\mathrm{f}}$ are plotted for $R e_{\mathrm{D}}$ up to $10^{7}$ comparing the two geometries here analyzed. The figure indicates that the lower the porosity, the higher the ratio $h_{\mathrm{i}} D / k_{\mathrm{f}}$, an effect which is less pronounced when computing the more "unobstructed" flow past the inlinearranged elliptic rods.

\subsection{3}

\section{Correlations for Laminar and Turbulent Flows}

Results for $h_{\mathrm{i}}$ covering both laminar [15] and turbulent flow regimes are plotted in Figure 1.13 along with correlation (1.51) by Kuwahara et al. (2001) [12]. Numerical results by Saito and de Lemos (2006) [38] using both low and high Re models are also plotted showing a good overlap of values calculated with both models around $R e_{\mathrm{D}}=6 \times 10^{4}$.

Figure 1.14 shows numerical results for the interfacial convective heat transfer coefficient for various porosities and plotted for $R e_{\mathrm{D}}$ up to $10^{7}$. In order to obtain a correlation for $h_{\mathrm{i}}$ in the turbulent regime, all curves were first collapsed after plotting them in terms of $R e_{\mathrm{D}} / \phi$, as shown in Figure 1.15. Next, the least squares technique was applied in order to determine the best correlation, which led to a minimum 

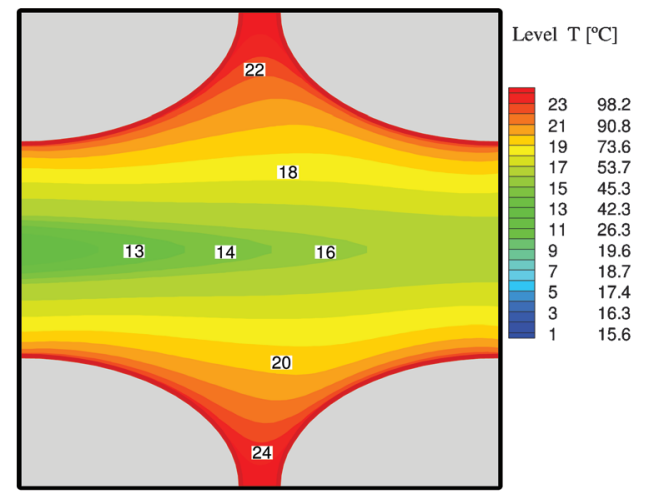

a) $R e_{D}=100$ and $\phi=0.60$
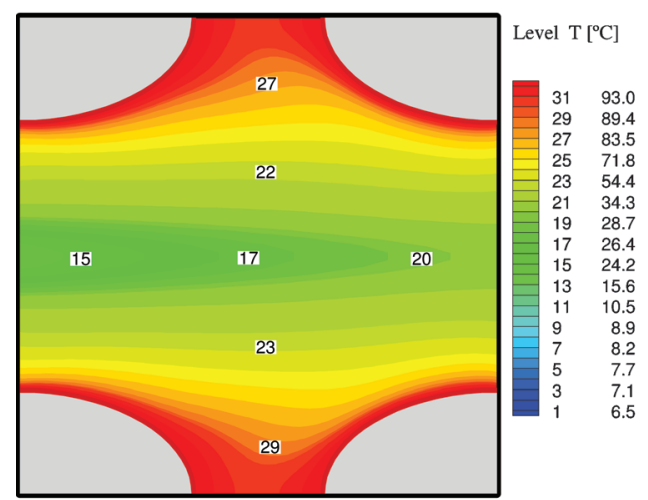

c) $R e_{D}=100$ and $\phi=0.75$

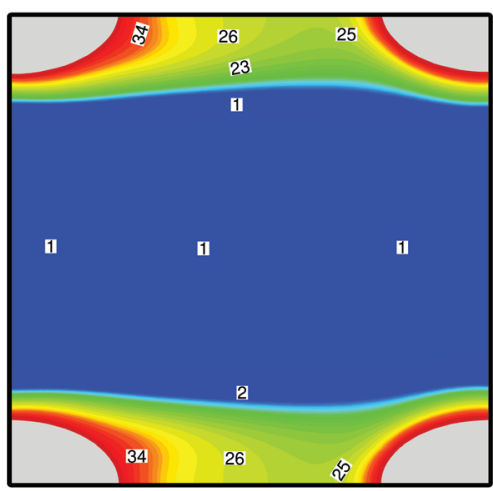

\begin{tabular}{|ll} 
Level & $\mathrm{T}\left[{ }^{\circ} \mathrm{C}\right]$ \\
\hline 35 & 91.9 \\
33 & 86.1 \\
31 & 83.6 \\
29 & 81.5 \\
27 & 77.6 \\
\hline 25 & 72.2 \\
\hline 23 & 68.0 \\
21 & 64.1 \\
19 & 61.6 \\
17 & 60.7 \\
15 & 60.1 \\
13 & 59.4 \\
11 & 58.6 \\
\hline 9 & 57.6 \\
\hline 7 & 56.5 \\
5 & 55.4 \\
3 & 54.4 \\
1 & 39.0 \\
\hline & \\
\hline
\end{tabular}

e) $R e_{D}=100$ and $\phi=0.90$

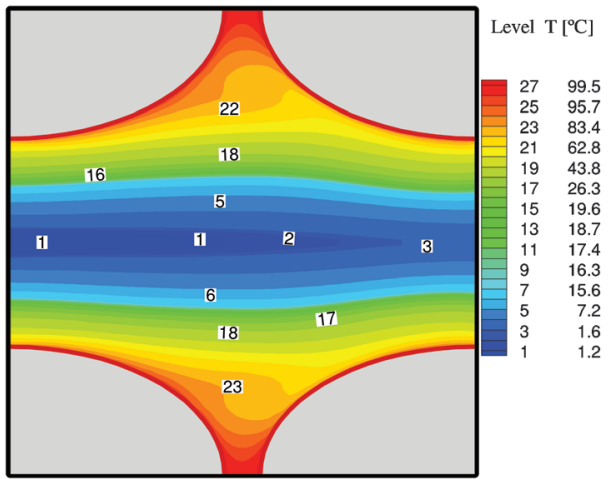

b) $R e_{D}=1000$ and $\phi=0.60$

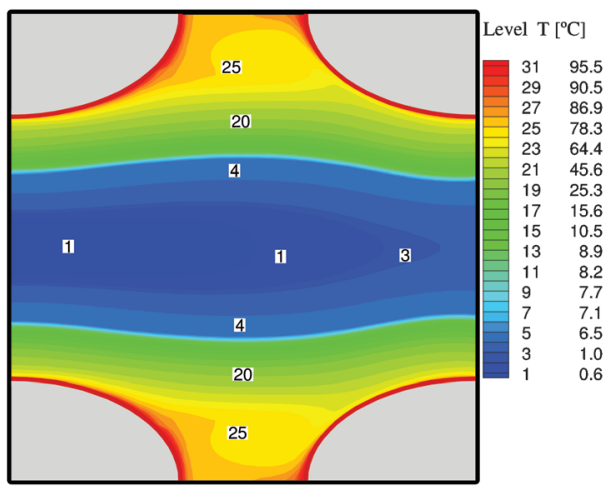

d) $R e_{D}=1000$ and $\phi=0.75$

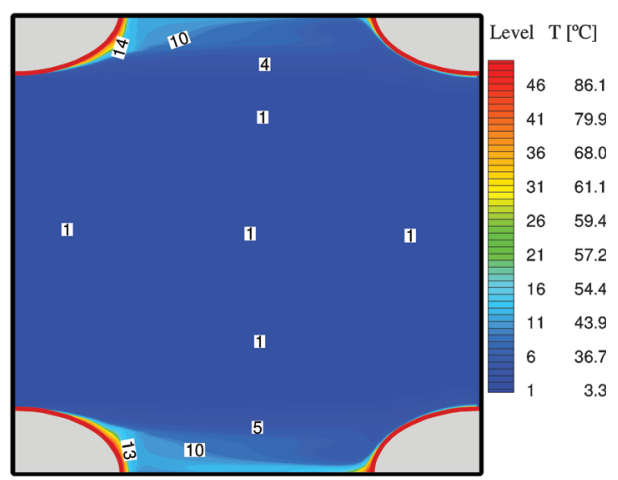

f) $R e_{D}=1000$ and $\phi=0.90$

Figure 1.7 Temperature fields in a periodic cell formed by elliptic rods. 
22 1 Interfacial Heat Transport in Highly Permeable Media
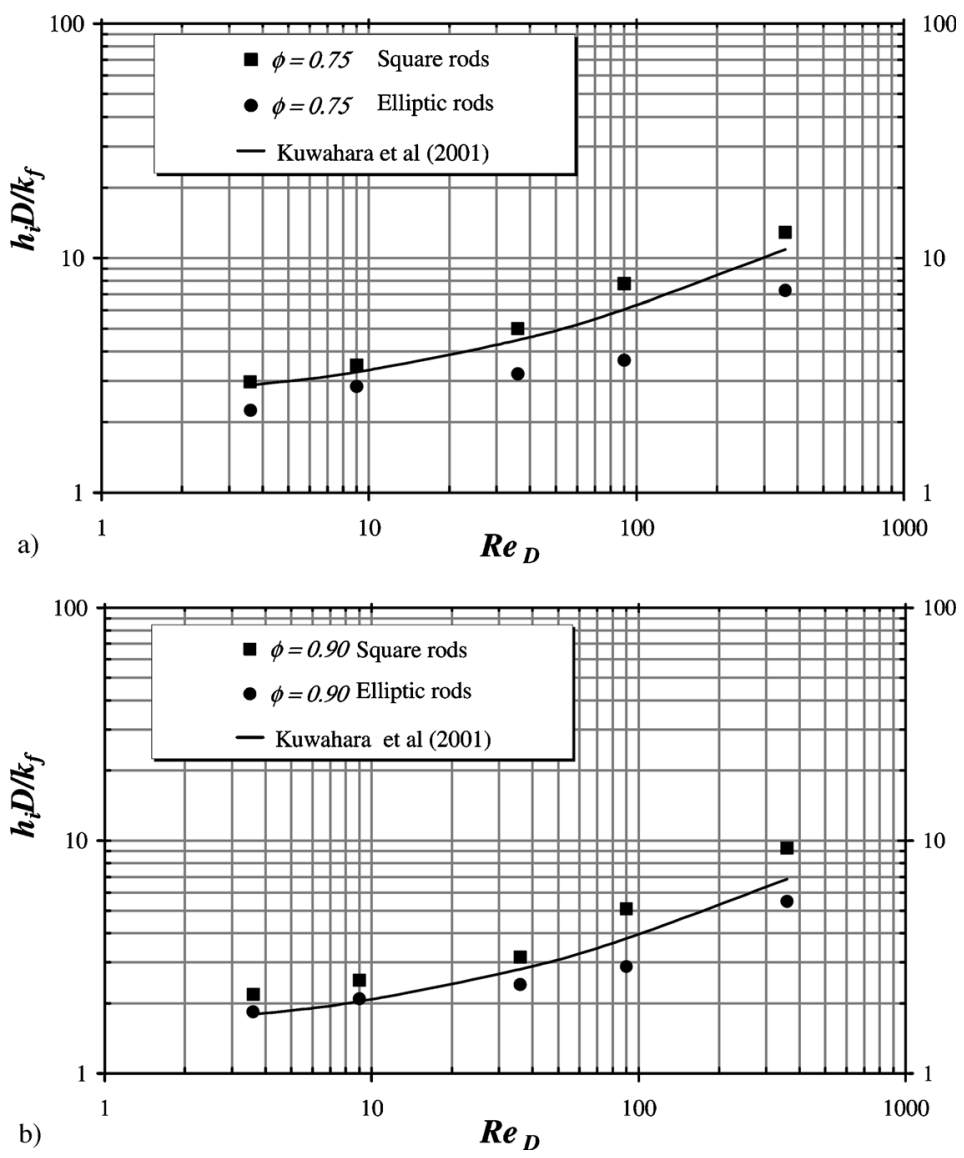

Figure 1.8 Comparison between square rods, elliptic rods and correlation of Kuwahara et al. [12] for $\phi=0.75$ and 0.90 .

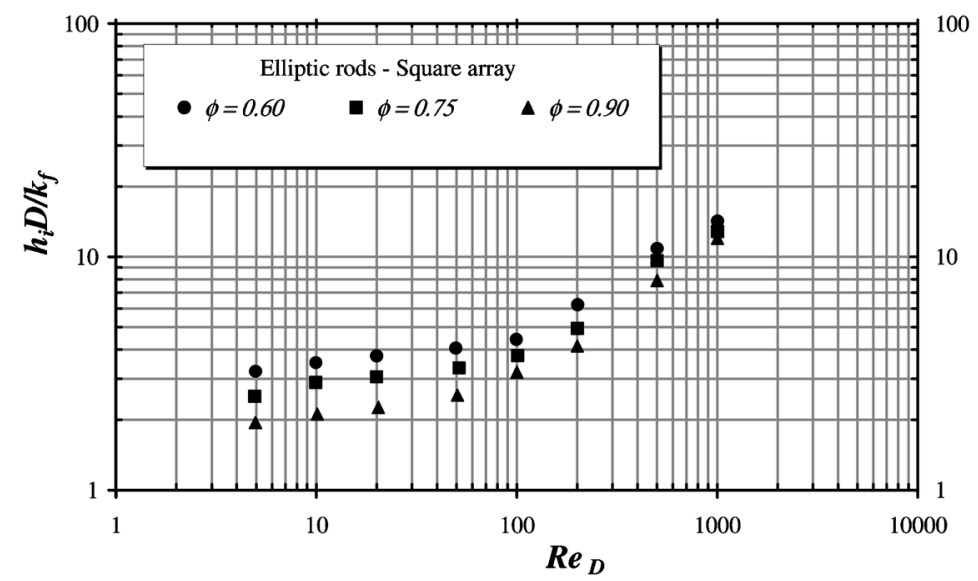

Figure 1.9 Effect of porosity $\phi$ on $h_{\mathrm{i}}$ for $\operatorname{Pr}=1$ and laminar flow. 
a)
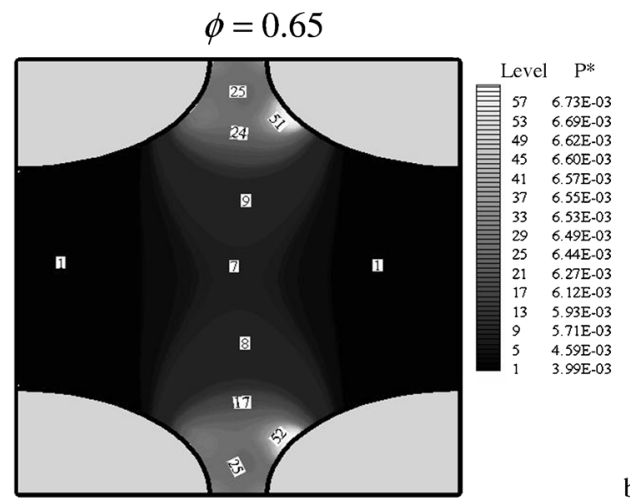

b)

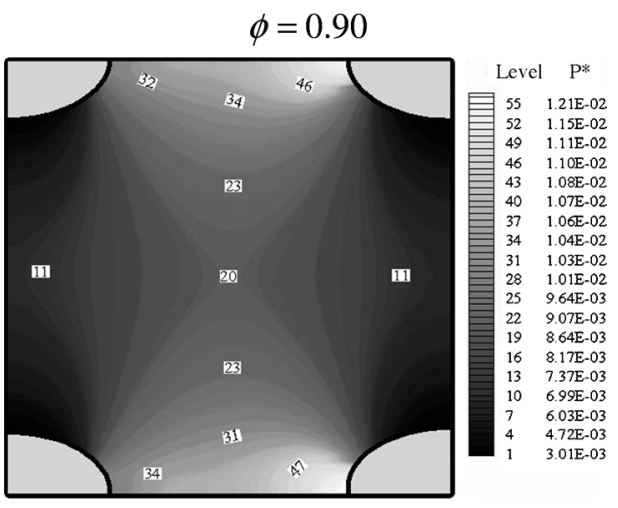

c)
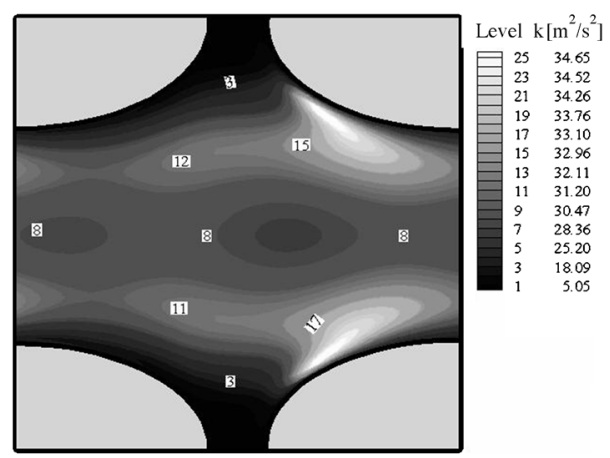

e)

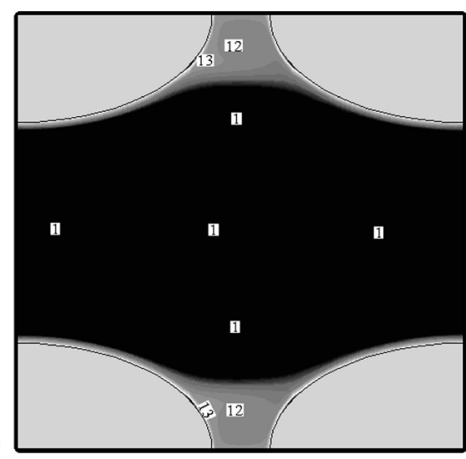

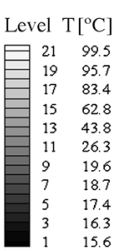

Figure 1.10 Results for elliptic rods, $R e_{\mathrm{D}}=10^{5}$ :

$(a, b)$ pressure; $(c, d)$ turbulent kinetic energy; $(e, f)$ temperature field.

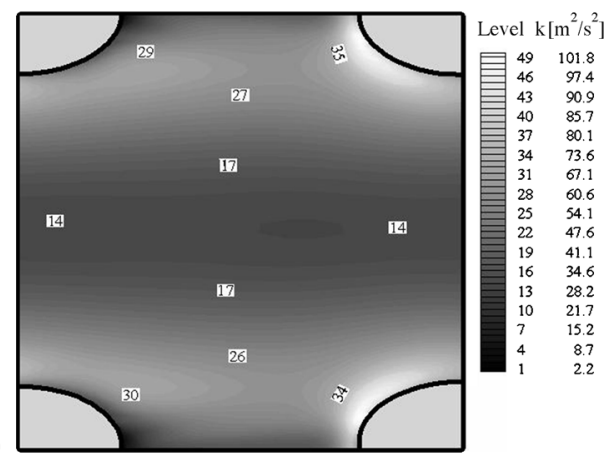

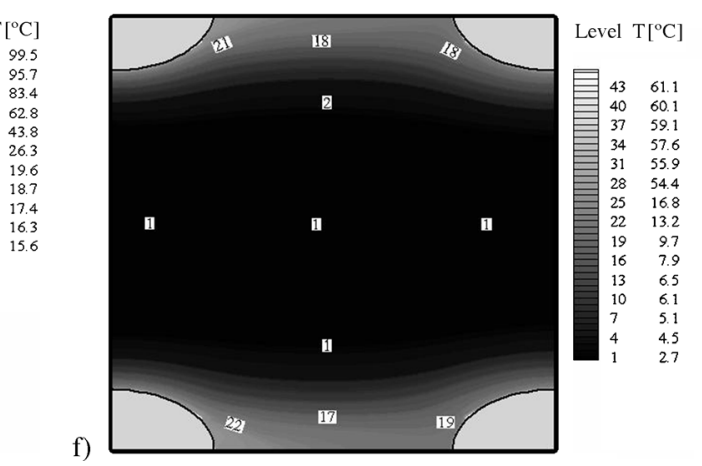

f) 


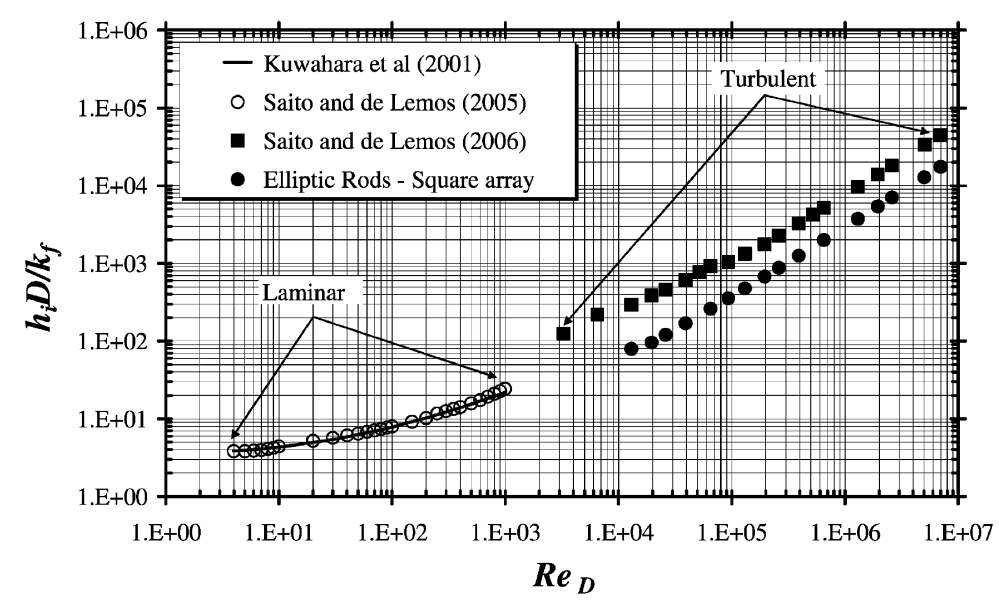

Figure 1.11 Effect of $R e_{\mathrm{D}}$ on $h_{\mathrm{i}}$ for turbulent flow, $\operatorname{Pr}=1$ and $\phi=0.65$ (data from $[12,15,38]$ ).

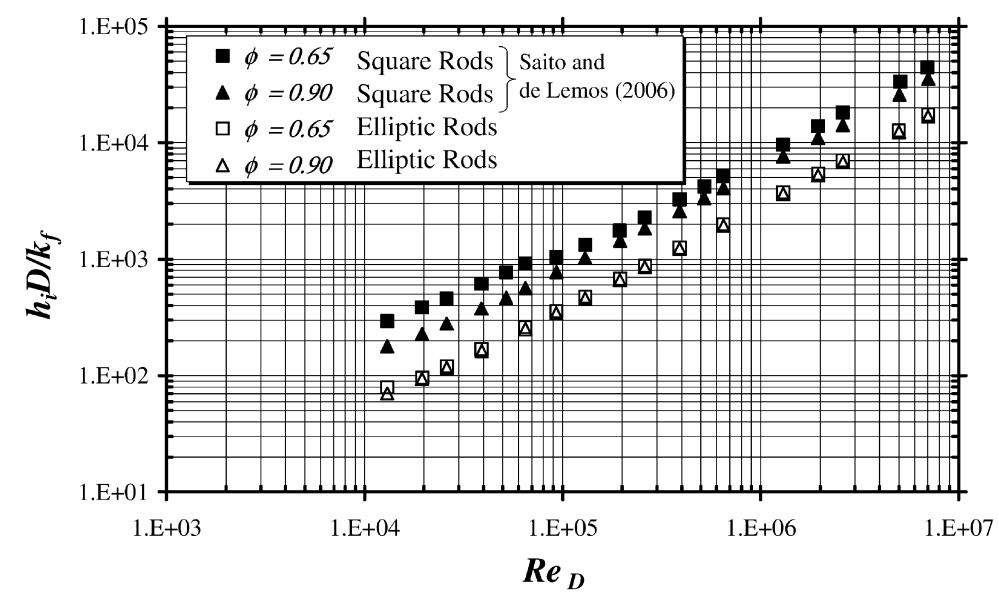

Figure 1.12 Effect of $R e_{\mathrm{D}}$ on $h_{\mathrm{i}}$ for distinct porosities and shapes (data from [38]).

overall error. Thus, the following expression was proposed in Saito and de Lemos (2006) [38]:

$$
\begin{aligned}
& \frac{h_{\mathrm{i}} D}{k_{\mathrm{f}}}=0.08\left(\frac{R e_{\mathrm{D}}}{\phi}\right)^{0.8} \operatorname{Pr}^{1 / 3} ; \text { for } 1.0 \times 10^{4}<\frac{R e_{\mathrm{D}}}{\phi}<2.0 \times 10^{7}, \\
& \quad \text { valid for } 0.2<\phi<0.9
\end{aligned}
$$

Equation (1.62), which gives the heat transfer coefficient for turbulent flow, is now compared with numerical results obtained with low and high Re models. Such a 


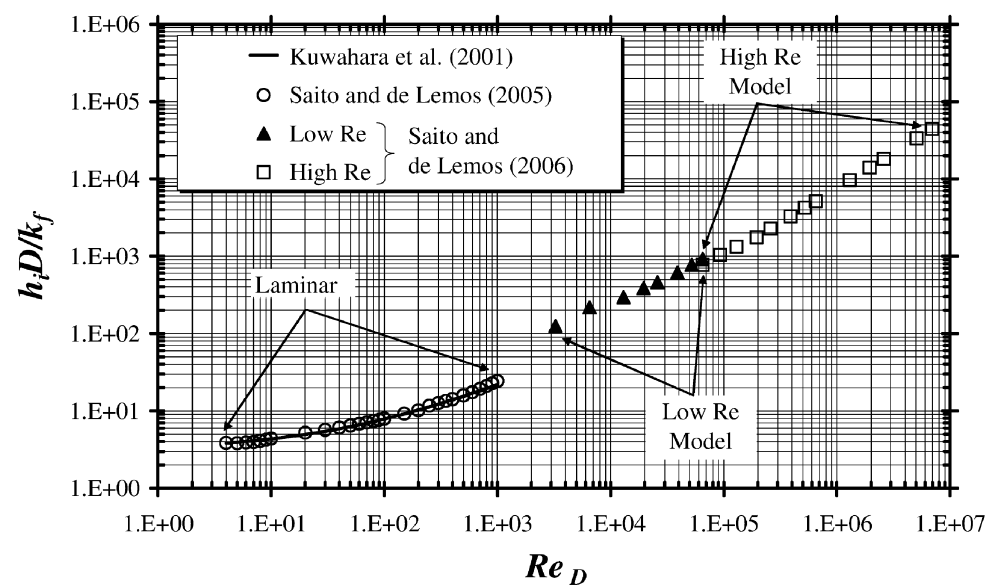

Figure 1.13 Effect of $R e_{\mathrm{D}}$ on $h_{\mathrm{i}}$ for turbulent flow, $\operatorname{Pr}=1$ and $\phi=0.65$ (data from $[12,15,38]$ ).

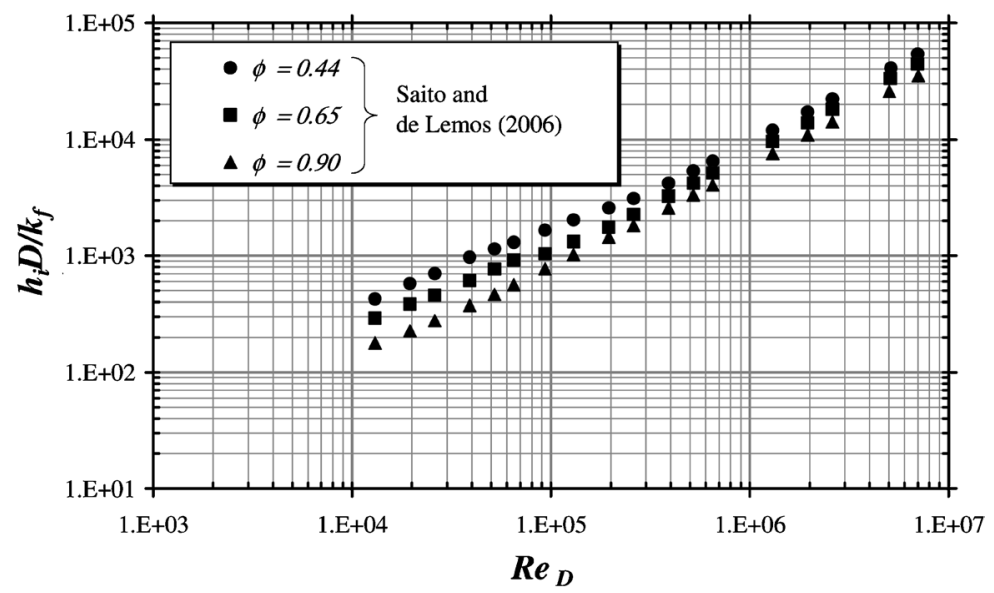

Figure 1.14 Effect of porosity on $h_{\mathrm{i}}$ for $\operatorname{Pr}=1$, square rods (data from [38]).

comparison is presented in Figure 1.16, which also shows computations using correlations given by Zhukauskas (1972) [57] and Wakao et al. (1979) [56], Eqs. (1.49) and (1.50), respectively. Table 1.2 summarizes correlations referred to in this chapter, through which the heat transfer coefficient $h_{\mathrm{i}}$ in porous media can be determined.

Finally, one can say that the agreement between the correlations in the literature and the numerical simulations here reported stimulates further investigation on this subject, contributing towards the building of a more general expression for the interfacial heat transfer coefficient for porous media. 


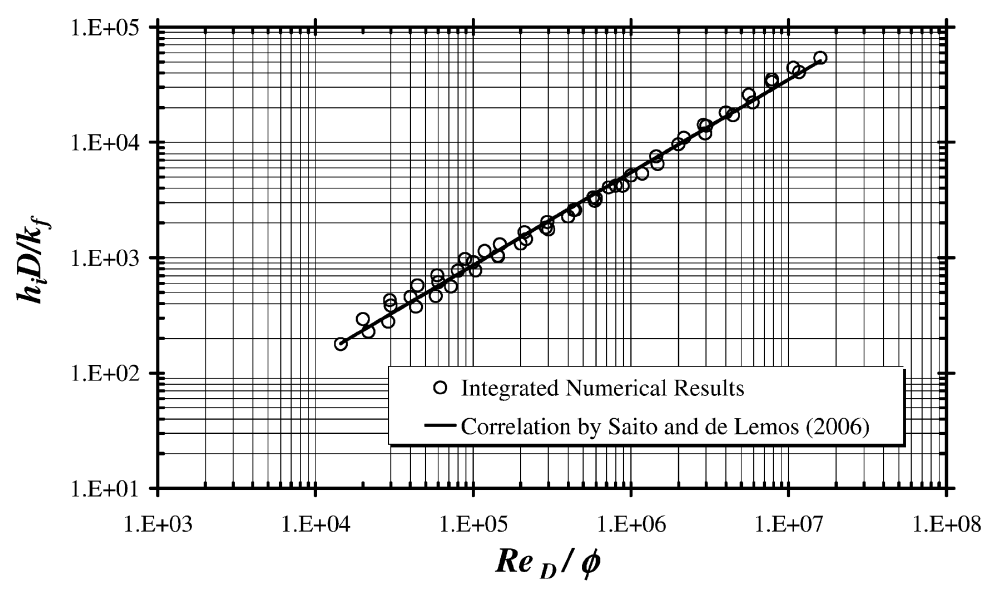

Figure 1.15 Scaled numerical results and comparison with correlation (1.62) by Saito and de Lemos [38].

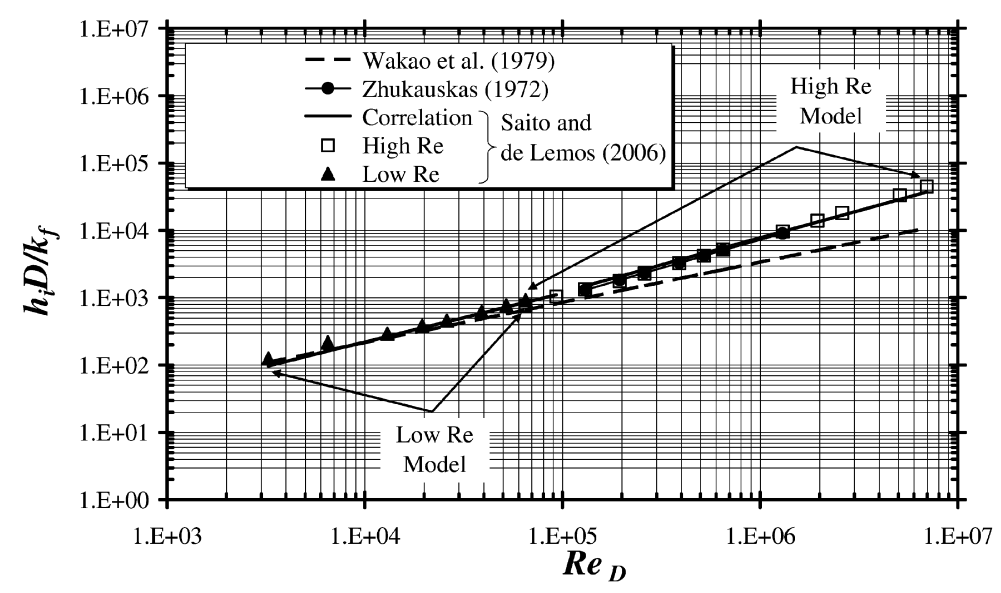

Figure 1.16 Comparison of integrated numerical results for $\phi=0.65$ and correlations from $[38,56,57]$.

Table 1.2 Correlations for interfacial heat transfer coefficient.

\begin{tabular}{llll}
\hline Correlation & Validity & Eq. & Ref. \\
\hline$\frac{h_{\mathrm{i}} D}{k_{\mathrm{f}}}=0.022 \operatorname{Re}_{\mathrm{D}}^{0.84} \operatorname{Pr}^{0.36}$ & $2 \times 10^{5}<R e_{\mathrm{D}}<2 \times 10^{6}$ & $(1.49)$ & {$[56]$} \\
$\frac{h_{\mathrm{i}} D}{k_{\mathrm{f}}}=2+1.1 \operatorname{Re}_{\mathrm{D}}^{0.6} \operatorname{Pr}^{1 / 3}$ & - & $(1.50)$ & {$[56]$} \\
$\frac{h_{\mathrm{i}} D}{k_{\mathrm{f}}}=\left(1+\frac{4(1-\phi)}{\phi}\right)+\frac{1}{2}(1-\phi)^{1 / 2} \operatorname{Re}_{\mathrm{D}} \operatorname{Pr}^{1 / 3}$ & Laminar flow, $0.2<\phi<0.9$ & $(1.51)$ & {$[12]$} \\
$\frac{h_{\mathrm{i}} D}{k_{\mathrm{f}}}=0.08\left(\frac{\operatorname{Re}_{\mathrm{D}}}{\phi}\right)^{0.8} \operatorname{Pr}^{1 / 3}$ & $\begin{array}{l}1.0 \times 10^{4}<R e_{\mathrm{D}} / \phi<2.0 \\
\times 10^{7} 0.2<\phi<0.9\end{array}$ & \\
\hline
\end{tabular}




\section{Conclusions}

A computational procedure for determining the convective coefficient of heat exchange between the porous substrate and the working fluid for a porous medium was detailed. As a result, macroscopically uniform laminar and turbulent flows through a periodic cell formed by square and elliptic rods were computed. Quantitative agreement was obtained when comparing laminar results herein with simulations by Kuwahara et al. (2001) [12]. For turbulent flows, low and high Reynolds turbulence models were employed in order to obtain the interfacial heat transfer coefficient. Correlations for determining the heat transfer coefficient were compared. Ultimately, it is expected that a more general correlation for $h_{\mathrm{i}}$ be obtained to be used in conjunction with more realistic two-energy equation models.

\section{Acknowledgments}

The authors thank CNPq and FAPESP, Brazil, for their invaluable financial support during the course of this and other research programs conducted at ITA.

\section{References}

$1 \mathrm{Hsu}$, C.T. and Cheng, P. (1990) Thermal dispersion in a porous medium. Int. $J$. Heat Mass Transfer, 33, 1587-1597.

2 Whitaker, S. (1966) Equations of motion in porous media. Chem. Eng. Sci., 21, 291-300.

3 Whitaker, S. (1967) Diffusion and dispersion in porous media. J. Am. Inst. Chem. Eng., 13, 420-427.

4 Bear, J. (1972) Dynamics of Fluids in Porous Media, American Elsevier, New York.

5 Nield, D.A. and Bejan, A. (1992) Convection in Porous Media, Springer, New York.

6 Ingham, D.B. and Pop, I. (1998) Transport Phenomena in Porous Media, Elsevier, Amsterdam, pp. 103-129.

7 Schumann, T.E.W. (1929) Heat transfer: liquid flowing through a porous prism. J. Franklin. Inst., 208, 405-416.

8 Quintard, M. (1998) Modeling local nonequilibrium heat transfer in porous media. in Proc. 11th Int. Heat Transfer
Conf., Kyongyu, Korea, vol. 1, 279-285.

9 Kaviany, M. (1995) Principles of Heat Transfer in Porous Media, 2nd edn, Springer, New York.

10 Quintard, M., Kaviany, M. and Whitaker, S. (1997) Two-medium treatment of heat transfer in porous media: numerical results for effective properties. $A d v$. Water Resour., 20, 77-94.

11 Ochoa-Tapia, J.A. and Whitaker, S. (1997) Heat transfer at the boundary between a porous medium and a homogeneous fluid. Int. J. Heat Mass Transfer, 40, 2691-2707.

12 Kuwahara, F., Shirota, M. and Nakayama, A. (2001) A numerical study of interfacial convective heat transfer coefficient in two-energy equation model for convection in porous media. Int. J. Heat Mass Transfer, 44, 1153-1159.

13 Nakayama, A., Kuwahara, F., Sugiyama, M. and Xu, G. (2001) A two-energy 
equation model for conduction and convection in porous media. Int. J. Heat Mass Transfer, 44, 4375-4379.

$14 \mathrm{Hsu}$, C.T. (1999) A closure model for transient heat conduction in porous media. J. Heat Transfer, 121, 733-739.

15 Saito, M.B. and de Lemos, M.J.S. (2005) Interfacial heat transfer coefficient for non-equilibrium convective transport in porous media. Int. Comm. Heat and Mass Transfer, 32, 667-677.

16 Gray, W.G. and Lee, P.C.Y. (1977) On the theorems for local volume averaging of multiphase system. Int. J. Multiphase Flow, 3, 333-340.

17 Pedras, M.H.J. and de Lemos, M.J.S. (2000) On the definition of turbulent kinetic energy for flow in porous media. Int. Comm. Heat and Mass Transfer, 27, 211-220.

18 Pedras, M.H.J. and de Lemos, M.J.S. (2001) Macroscopic turbulence modeling for incompressible flow through undeformable porous media. Int. J. Heat and Mass Transfer, 44, 1081-1093.

19 Pedras, M.H.J. and de Lemos, M.J.S. (2001) Simulation of turbulent flow in porous media using a spatially periodic array and a low-Re two-equation closure. Numer. Heat Transfer A: Applications, 39, 35-59.

20 Pedras, M.H.J. and de Lemos, M.J.S. (2001) On the mathematical description and simulation of turbulent flow in a porous medium formed by an array of ellipticrods. J. Fluids Eng., 123, 941-947.

21 Pedras, M.H.J. and de Lemos, M.J.S. (2003) Computation of turbulent flow in porous media using a Low Reynolds $k-\varepsilon$ model and an infinite array of transversally displaced elliptic rods. Numer. Heat Transfer A: Applications, 43, 585-602.

22 de Lemos, M.J.S. (2005) Fundamentals of the double-decomposition concept for turbulent transport in permeable media. Materialwissenschaft und Werkstofftechnik, 36, 586-593.
23 Rocamora F.D. Jr and de Lemos, M.J.S. (2000) Analysis of convective heat transfer of turbulent flow in saturated porous media. Int. Comm. Heat Mass Transfer, 27, 825-834.

24 de Lemos, M.J.S. and Rocamora, F.D. (2002) Turbulent transport modeling for heated flow in rigid porous media. Proc. 12th International Heat Transfer Conf. Grenoble, France, 18-23 August, 791-795.

25 de Lemos, M.J.S. and Braga, E.J. (2003) Modeling of turbulent natural convection in saturated rigid porous media. Int. Comm. Heat Mass Transfer, 30, 615-624.

26 Braga, E.J. and de Lemos, M.J.S. (2004) Turbulent natural convection in a porous square cavity computed with a macroscopic $k-\varepsilon$ model. Int. J. Heat and Mass Transfer, 47, 5639-5650.

27 Braga, E.J. and de Lemos, M.J.S.( (2005) Heat transfer in enclosures having a fixed amount of solid material simulated with heterogeneous and homogeneous models. Int. J. Heat Mass Transfer, 48, 4748-4765.

28 Braga, E.J. and de Lemos, M.J.S.( (2005) Laminar natural convection in cavities filled with circular and square rods. Int. Comm. Heat Mass Transfer, 32, 1289-1297.

29 Braga, E.J. and de Lemos, M.J.S. (2006) Turbulent heat transfer in an enclosure with a horizontal porous plate in the middle. J. Heat Transfer, 128, 1122-1129.

30 Braga, E.J. and de Lemos, M.J.S. (2006) Simulation of turbulent natural convection in a porous cylindrical annulus using a macroscopic twoequation model. Int. J. Heat Mass Transfer, 49, 4340-4351.

31 de Lemos, M.J.S. and Mesquita, M.S. (2003) Turbulent mass transport in saturated rigid porous media. Int. Comm. Heat Mass Transfer, 30, 105-113.

32 de Lemos, M.J.S. and Tofaneli, L.A. (2004) Modeling of double-diffusive turbulent natural convection in porous media. Int. J. Heat Mass Transfer, 47, 4221-4231. 
33 de Lemos, M.J.S. and Pedras, M.H.J. (2001) Recent mathematical models for turbulent flow for saturated rigid porous media. J. Fluids Eng., 123, 935-940.

34 Silva, R.A. and de Lemos, M.J.S. (2003) Numerical analysis of the stress jump interface condition for laminar flow over a porous layer. Numer. Heat Transfer A: Applications, 43, 603-617.

35 Silva, R.A. and de Lemos, M.J.S. (2003) Turbulent flow in a channel occupied by a porous layer considering the stress jump at the interface. Int. J. Heat Mass Transfer, 46, 5113-5121.

36 de Lemos, M.J.S. (2005) Turbulent kinetic energy distribution across the interface between a porous medium and a clear region. Int. Comm. Heat Mass Transfer, 32, 107-115.

37 de Lemos, M.J.S. and Silva, R.A. (2006) Turbulent flow over a layer of a highly permeable medium simulated with a diffusion-jump model for the interface. Int. J. Heat Mass Transfer, 49, 546-556.

38 Saito, M.B. and de Lemos, M.J.S. (2006) A correlation for interfacial heat transfer coefficient for turbulent flow over an array of square rods. J. Heat Transfer, 128, 444-452.

39 de Lemos, M.J.S. (2006) Turbulence in Porous Media: Modeling and Applications, Elsevier, New York.

40 Launder, B.E. and Spalding, D.B. (1974) The numerical computation of turbulent flows. Comp. Meth. Appl. Mech. Eng., 3, 269-289.

41 Masuoka, T. and Takatsu, Y. (1996) Turbulence model for flow through porous media. Int. J. Heat Mass Transfer, 39, 2803-2809.

42 Kuwahara, F., Nakayama, A. and Koyama, H. (1996) A numerical study of thermal dispersion in porous media. $J$. Heat Transfer, 118, 756-761.

43 Kuwahara, F. and Nakayama, A. (1998) Numerical modeling of non-Darcy convective flow in a porous medium.
Heat Transfer 1998: Proc. 11th Int. Heat Transfer Conf. Kyongyu, Korea, Taylor \& Francis, Washington, DC, 4, 411-416.

44 Kuwahara, F., Kameyama, Y., Yamashita, S. and Nakayama, A. (1998) Numerical modeling of turbulent flow in porous media using a spatially periodic array. $J$. Porous Media, 1, 47-55.

45 Ergun, S. (1952) Fluid flow through packed columns. Chem. Eng. Pro., 48, 89-94.

46 Nakayama, A. and Kuwahara, F. (1999) A macroscopic turbulence model for flow in a porous medium. J. Fluids Eng., 121, 427-433.

47 Lee, K. and Howell, J.R. (1987) Forced convective and radiative transfer within a highly porous layer exposed to a turbulent external flow field. Proc. 1987 ASME-JSME Thermal Engineering Joint Conf. Honolulu, HI, ASME, New York, 2, 377-386.

48 Antohe, B.V. and Lage, J.L. (1997) A general two-equation macroscopic turbulence model for incompressible flow in porous media. Int. J. Heat Mass Transfer, 40, 3013-3024.

49 Getachewa, D., Minkowycz, W.J. and Lage, J.L. (2000) A modified form of the $k-\varepsilon$ model for turbulent flow of a incompressible fluid in porous media. Int. J. Heat Mass Transfer, 43, 2909-2915.

50 de Lemos, M.J.S. (2005) Mathematical Modeling and Applications of Turbulent Heat and Mass Transfer in Porous Media. in Handbook of Porous Media, $2^{\text {nd }}$ ed. (ed.V. Kambiz ), Taylor \& Francis Group, LLC, New York, pp. 409-454. Chapter 10

51 de Lemos, M.J.S. (2005) The doubledecomposition concept for turbulent transport in porous media. in Transport Phenomena in Porous Media III (ed. D.B. Ingham and I. Pop), Elsevier Science, Amsterdam, pp. 1-33. 
52 de Lemos, M.J.S. (2004) Turbulent heat and mass transfer in porous media. inEmerging Technologies and Techniques in Porous Media (ed. D.B. Ingham, A. Bejan ,E. Mamut andI. Pop), Kluwer Academic, Dordrecht, vol. 134, pp. 157-168.

53 Lage, J.L., de Lemos, M.J.S. and Nield, D.A. (2002) Modeling turbulence in porous media. inTransport Phenomena in Porous Media II (ed. D.B. Ingham and I. Pop), Pergamon Press, Amsterdam, pp. 198-230.

54 Slattery, J.C. (1967) Flow of viscoelastic fluids through porous media. AIChE J., 13, 1066-1071.
55 Forchheimer, P. (1901) Wasserbewegung durch Boden. Z. Ver. Deutsch. Ing., 45, 1782-1788.

56 Zhukauskas, A. (1972) Heat transfer from tubes in cross flow. Advances in Heat Transfer 8, Academic Press, New York.

57 Wakao, N., Kaguei, S. and Funazkri, T. (1979) Effect of fluid dispersion coefficients on particle-to-fluid heat transfer coefficients in packed bed. Chem. Eng. Sci., 34, 325-336.

58 Patankar, S.V. (1980) Numerical Heat Transfer and Fluid Flow, Hemisphere, Washington, DC. 